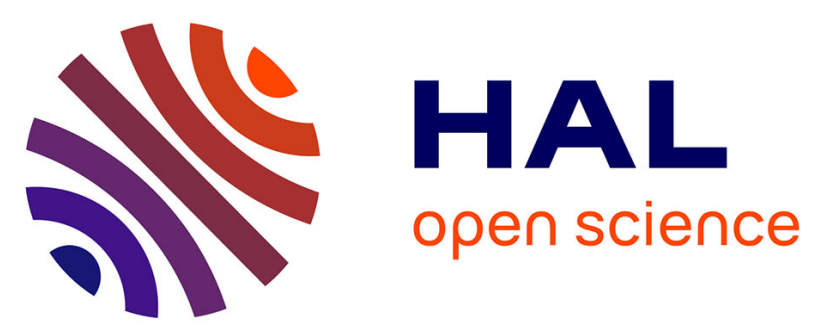

\title{
Stratified Inertial Subrange Inferred from In Situ Measurements in the Bottom Boundary Layer of the Rockall Channel
}

Pascale Bouruet-Aubertot, Hans van Haren, Marie-Pascale Lelong

\section{To cite this version:}

Pascale Bouruet-Aubertot, Hans van Haren, Marie-Pascale Lelong. Stratified Inertial Subrange Inferred from In Situ Measurements in the Bottom Boundary Layer of the Rockall Channel. Journal of Physical Oceanography, 2010, 40, pp.2401-2417. 10.1175/2010JPO3957.1 . hal-00753830

\section{HAL Id: hal-00753830 \\ https://hal.science/hal-00753830}

Submitted on 3 Nov 2021

HAL is a multi-disciplinary open access archive for the deposit and dissemination of scientific research documents, whether they are published or not. The documents may come from teaching and research institutions in France or abroad, or from public or private research centers.
L'archive ouverte pluridisciplinaire HAL, est destinée au dépôt et à la diffusion de documents scientifiques de niveau recherche, publiés ou non, émanant des établissements d'enseignement et de recherche français ou étrangers, des laboratoires publics ou privés.

\section{(c)(1)}

Distributed under a Creative Commons Attribution| 4.0 International License 


\title{
Stratified Inertial Subrange Inferred from In Situ Measurements in the Bottom Boundary Layer of the Rockall Channel
}

\author{
PAscale Bouruet-Aubertot \\ LOCEAN/UPMC, Paris, France \\ HANS VAN HAREN \\ Royal Netherland Institute for Sea Research (NIOZ), Texel, Netherlands \\ M. PASCALE LELONG \\ North West Research Associates, Seattle, Washington
}

(Manuscript received 27 November 2007, in final form 8 June 2010)

\begin{abstract}
Deep-ocean high-resolution moored temperature data are analyzed with a focus on superbuoyant frequencies. A local Taylor hypothesis based on the horizontal velocity averaged over $2 \mathrm{~h}$ is used to infer horizontal wavenumber spectra of temperature variance. The inertial subrange extends over fairly low horizontal wavenumbers, typically within $2 \times 10^{-3}$ and $2 \times 10^{-1}$ cycles per minute (cpm). It is therefore interpreted as a stratified inertial subrange for most of this wavenumber interval, whereas in some cases the convective inertial subrange is resolved as well. Kinetic energy dissipation rate $\epsilon$ is inferred using theoretical expressions for the stratified inertial subrange. A wide range of values within $10^{-9}$ and $4 \times 10^{-7} \mathrm{~m}^{2} \mathrm{~s}^{-3}$ is obtained for time periods either dominated by semidiurnal tides or by significant subinertial variability. A scaling for $\epsilon$ that depends on the potential energy within the inertio-gravity waves (IGW) frequency band $\mathrm{PE}_{\mathrm{IGW}}$ and the buoyancy frequency $N$ is proposed for these two cases. When semidiurnal tides dominate, $\epsilon \simeq\left(\mathrm{PE}_{\mathrm{IGW}} N\right)^{3 / 2}$, whereas $\epsilon \simeq \mathrm{PE}_{\mathrm{IGW}} N$ in the presence of significant subinertial variability. This result is obtained for energy levels ranging from 1 to 30 times the Garrett-Munk energy level and is in contrast with classical finescale parameterization in which $\epsilon \sim\left(\mathrm{PE}_{\mathrm{IGW}}\right)^{2}$ that applies far from energy sources. The specificities of the stratified bottom boundary layer, namely a weak stratification, may account for this difference.
\end{abstract}

\section{Introduction}

Large-scale down to submesoscale variability in the ocean is strongly influenced by the earth's rotation and vertical density stratification. However, at smaller scales these influences become negligible and the dynamics becomes isotropic while the classical spectral shapes of $3 \mathrm{D}$ turbulence are recovered. In terms of frequency $\omega$, both rotation and stratification are expected to play a role at least for the range $\omega<N$, with most of the energy carried by inertio-gravity waves (IGW) with $\omega \in[f, N]$ ( $f$ being the inertial frequency and $N$ being the buoyancy frequency) when $N \gg f$. The energy sources for these waves, namely

Corresponding author address: Pascale Bouruet-Aubertot, LOCEAN/UPMC, BP100, T45-55 5E, 4 place Jussieu, 75252 Paris CEDEX 05, France.

E-mail: pascale.bouruet-aubertot@locean-ipsl.upmc.fr tidal and atmospheric forcing, are significant (e.g., Munk and Wunsch 1998). As a result, IGW constitute an important source of turbulence when they break. The manner in which energy is transferred from large scales to small dissipative scales is an important issue for the parameterization of kinetic energy dissipation and mixing.

In the case of low energy levels corresponding to the Garrett-Munk model (Garrett and Munk 1972, hereinafter GM72), vertical wavenumber spectra inferred from observations in the main thermocline show that there is an intermediate spectral range, a stratified inertial turbulent subrange also referred to as "buoyancy subrange" or "saturated IGW" subrange, between the IGW domain and that of small-scale turbulence (e.g., Gregg 1987). This stratified inertial turbulent subrange corresponds to strongly nonlinear saturated waves, vortical modes, and turbulent motions with vertical Froude numbers $W /(N H) \sim 1$, where $W$ is typical vertical velocity, $N$ is 

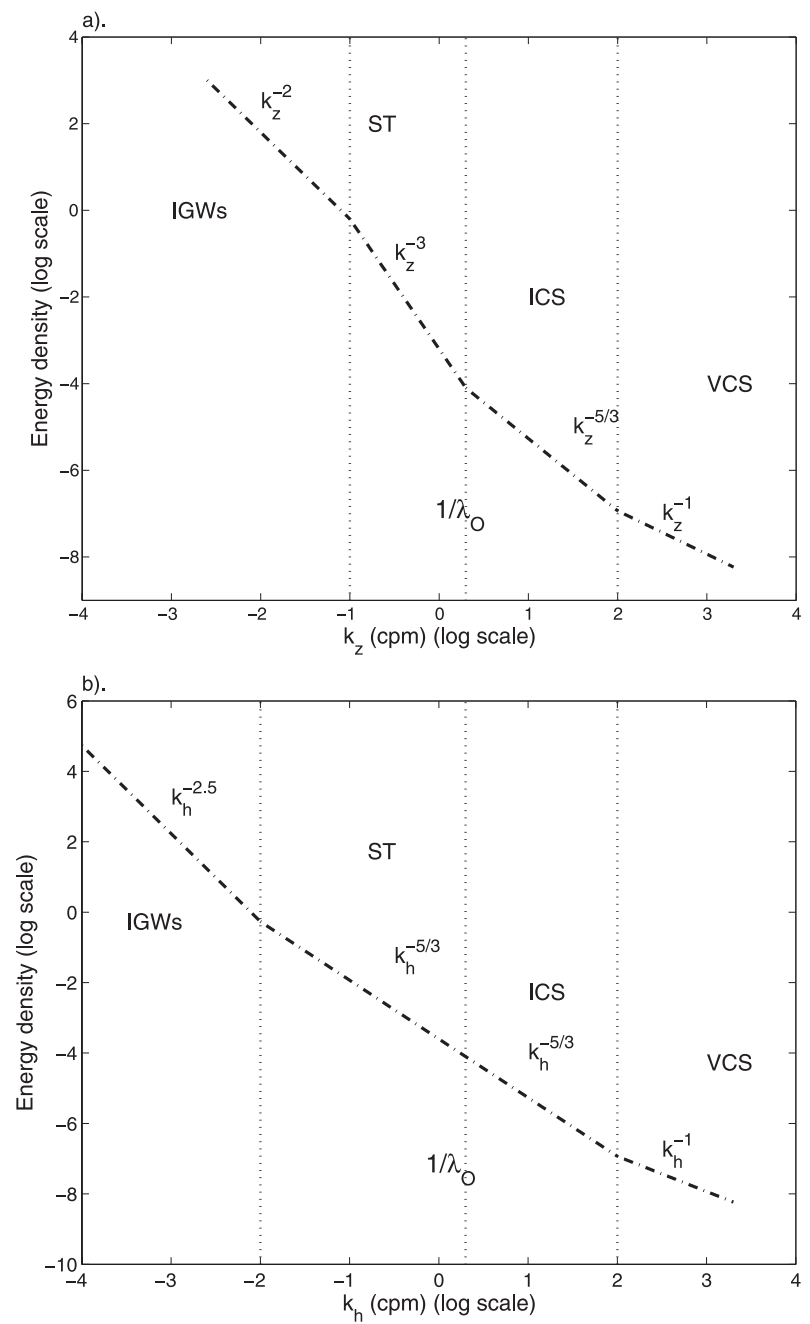

FIG. 1. Typical shape of spectra of temperature variance $E_{\theta}$ in the oceanic thermocline as a function of (a) vertical wavenumber $k_{z}$ and (b) horizontal wavenumber $k_{h}$. (a) At small vertical wavenumbers, the spectral slope of $E_{\theta}$ is close to -2, in agreement with the GM72 model for weakly nonlinear IGW $\left(\mathrm{IGW}_{s}\right)$. The STs, also referred to as saturated IGWs, are characterized by a -3 spectral slope. In the ICS, the slope is equal to $-5 / 3$ and to -1 in the viscous convective subrange (VCS). (b) At small horizontal wavenumbers, the slope is about -2.5 (Klymak and Moum 2007a), at intermediate horizontal wavenumber an inertial range with a $-5 / 3$ slope in which two parts can be distinguished is observed: a stratified inertial subrange for wavenumbers smaller than the Ozmidov wavenumber $k_{O}$ and an inertial convective subrange for higher horizontal wavenumbers. The wavelength that corresponds to the transition between the ST and the ICS is the Ozmidov scale, $\lambda_{O}=\sqrt{\epsilon / N^{3}}$.

buoyancy frequency and $H$ is typical vertical length scale (e.g., Polzin et al. 2003; Lindborg 2006; Riley and Lindborg 2008). The shape of vertical wavenumber temperature variance spectra follows a power law with an exponent equal to -2 in the IGW domain, -3 in the stratified turbulence subrange, and $-5 / 3$ in the inertial-convective subranges (e.g., Gregg 1977; see Fig. 1a). In the case of high energy levels relative to GM72 levels, the question of the existence of the stratified turbulence subrange is raised because the Ozmidov scale $\lambda_{O} \sqrt{\epsilon / N^{3}}$, which is the transition scale between this subrange and the inertial subrange, increases with increasing energy level. There is some observational evidence from the atmosphere (e.g., Alisse and Sidi 2000) that temperature variance spectra display a -3 slope in "calm regions" and a $-5 / 3$ slope in "turbulent layers."

Only a few observations in the ocean allow the characterization of horizontal wavenumber spectra (e.g., McKean and Ewart 1974; Ewart 1976; Katz and Briscoe 1979; Dugan et al. 1986; Klymak and Moum 2007b). In the aforementioned Klymak and Moum study, evidence of an inertial subrange, extending to horizontal wavenumbers $k_{h}$, smaller than the Ozmidov wavenumber $k_{O}$ by at least one or two orders of magnitude, is noted. An interpretation pointed out by Riley and Lindborg (2008) is that the lowest part of this inertial subrange, $k_{h}<k_{O}$, cannot be an inertial convective subrange but rather a stratified inertial subrange. Indeed, stratification effects are dominant, allowing a downward energy cascade and horizontal and vertical wavenumber spectra exhibit different behavior (Lindborg 2006). Note that this downward energy cascade does not exclude the possibility of an inverse energy cascade as shown by Riley and de Bruyn Kops (2003) in their simulations. In this stratified turbulence, the horizontal Froude number $U /(N L)<1$, where $U$ refers to typical horizontal velocity and $L$ refers to typical horizontal length scale; the vertical Froude number is of order 1; and the aspect ratio of vertical to horizontal wavelengths is small (e.g., Riley and Lindborg 2008). For lower horizontal wavenumbers, a slope of about -2.5 is observed for the ocean. A slightly steeper slope of -3 is reported in the atmosphere (e.g., Nastrom and Gage 1985).

The main focus of this paper is on the characterization of temperature variance spectra as a function of the IGW energy level and stratification, using high-resolution temperature observations from the deep ocean. First, we investigate how the shape of the temperature variance spectra evolves as a function of the dynamics at larger scales. Second, the scaling for energy dissipation as a function of $\mathrm{PE}_{\mathrm{IGW}}$ is inferred.

The paper is organized as follows: In the first section, the dataset is described and the general circulation of water masses in the Rockall Channel is introduced; an overview of the time evolution of the frequency content at subbuoyant frequencies is then given, which allows the delineation of different dynamical regimes. Temperature variance frequency spectra focused on superbuoyant frequencies are analyzed in section 3. Kinetic energy dissipation is then inferred from the inertial subrange evidenced 
in horizontal wavenumber spectra. The formation of sheets is eventually examined in section 4 . Results are discussed in the last section.

\section{Data}

Data were collected as part of the Royal Netherland Institute for Sea Research (NIOZ) Rockall Channel Studies (ROCS) program, mainly aimed at the study of sediment resuspension dynamics on the southeastern slope of Rockall Channel (Bonnin et al. 2006, hereinafter B2006). Moorings were deployed at the foot of the continental slope between 18 July and 31 August 2002 (Fig. 2). Our analysis focuses on the dynamics at high frequencies using measurements from the mooring located just on the abyssal plain at $2975 \mathrm{~m}$ at $\left(54^{\circ} 10^{\prime} \mathrm{N}\right.$, $\left.14^{\circ} 01^{\prime} \mathrm{W}\right)$. This mooring was equipped with a $300-\mathrm{kHz}$ RD Instruments (RDI) acoustic Doppler current profiler (ADCP) looking upward from a bottom lander, to which a high-resolution NIOZ-built thermistor string was attached. The ADCP sampled eighty 1-m vertical bins every $15 \mathrm{~s}$. The thermistor string consisted of 32 sensors spaced at $0.5-\mathrm{m}$ intervals within the depth range $2893.5-2910 \mathrm{~m}$ that is between 66 and $81.5 \mathrm{~m}$ above the sea floor. Its sampling interval was $30 \mathrm{~s}$. The sensors can be extremely precise ( $<0.1 \mathrm{~m} \mathrm{~K}$; van Haren et al. 2001), but absolute accuracy depends on in situ calibration $\sim(1-2) \mathrm{m} \mathrm{K}$. Temperature time series were analyzed over the whole depth range 2893.5-2910.0 m, but the vertical structure could only be inferred from the first nine sensors because of a lack of absolute accuracy of a few sensors below. Adequate ADCP data for statistical analysis of IGW were available within the depth range $2920 \mathrm{~m}-2968 \mathrm{~m}$, which is $10 \mathrm{~m}$ deeper than the deepest thermistor. In addition, four CTD profiles were performed near the moorings during deployment and recovery. As far as could be judged from these profiles, the temperature-salinity (TS) relationship was reasonably tight (Fig. 3).

\section{Variability at subbuoyant frequencies}

The variability at subbuoyant frequencies is first investigated to identify the different dynamical regimes. Time series and wavelet transforms of temperature and horizontal velocities are displayed in Figs. 4, 5, and 6. The wavelet transform is shown over two frequency ranges. The first range corresponds to frequencies within $1.38 \times 10^{-2}$ and $1.67 \times 10^{-1} \mathrm{cph}$, equivalent to periods within 72 and $6 \mathrm{~h}$. The second range corresponds to frequencies within $5.56 \times 10^{-2}$ and $9.74 \times 10^{-1} \mathrm{cph}$, with corresponding periods within 18 and $1 \mathrm{~h}$. This second frequency range includes the IGW frequency range because $N \sim(7.45 \pm 2.29) \times 10^{-1} \mathrm{cph}$ and the inertial frequency is equal to $6.76 \times 10^{-2} \mathrm{cph}$, equivalent to an inertial period $T_{f}=14.8 \mathrm{~h}$. The buoyancy frequency is estimated from the vertical temperature gradient between the first and last sensors of the thermistor chain; that is, for a 16-m vertical separation, $N^{2}=g \alpha(\Delta T / \Delta z)_{16 \mathrm{~m}}$, where $\alpha$ is the thermal expansion coefficient, $\alpha \simeq 1.5710^{-4}{ }^{\circ} \mathrm{C}^{-1}$ (Fig. 4a). CTD data were used to establish the TS relationship and hence to include the effects of salinity on $N$. The ratio $N / f \sim O(10)$ is relatively small in comparison with near-surface pycnoclines because of a fairly weak mean stratification.

The dynamics is mostly driven by semidiurnal tides as shown in Fig. 5. We focus, in the following discussion, on the wavelet transform of temperature that gives evidence of different regimes. During the first 21 days, until day 220 , most of the variability lies within two frequency bands, the semidiurnal band $M_{2}$ and, to a lesser extent, the near-inertial band (Figs. 4c,d). Some variability at higher frequencies most evident in the vertical temperature gradient across $\Delta z=16 \mathrm{~m}$ can be noticed (Fig. 4d). The direction of the mean horizontal current (averaged over two tidal periods) varies weakly, directed eastward (Fig. 6a), and the mean stratification is statically stable (Fig. 4a). The dynamical regime, represented by this period of 21 days, is called the IGW regime.

During the following 15 days (220-235 days), the distribution of energy in frequency space differs significantly: the subinertial variability is strong, with two components centered around periods equal to 60 and $40 \mathrm{~h}$ (Fig. 4b), and the energy is widely distributed in the higher frequency band, corresponding to periods within 6 and $18 \mathrm{~h}$ (Fig. 4c). This drastic change in temperature variability at subinertial frequencies is correlated with a change in the current direction as shown in Fig. 6. Between days 220 and 230 , the current that was initially directed eastward along the isobaths rotates toward the south-southwest, with a dominant cross-slope component (Fig. 6a). During this period, vigorous gravity currents and solibores occasionally move up and down the slope (B2006). The buoyancy frequency is still within the same range of 0.5 to $1 \mathrm{cph}$. This second dynamical regime will be called the subinertial event (SIE) and IGW (SIE-IGW) regime.

Next, from day 235 until day 244, the stratification weakens until it becomes neutral around days 239-241. The mean current still has a significant cross-slope component (Fig. 6a). Most of the variability lies within the superinertial frequency band (Figs. $4 b, c)$. The variability in $\Delta T / \Delta z$ is small in comparison with the previous regimes, a consequence of the almost neutral stratification (Fig. 4e). This last regime will be called the weakly stratified to neutral (WSN) regime. 
Longitude (W)
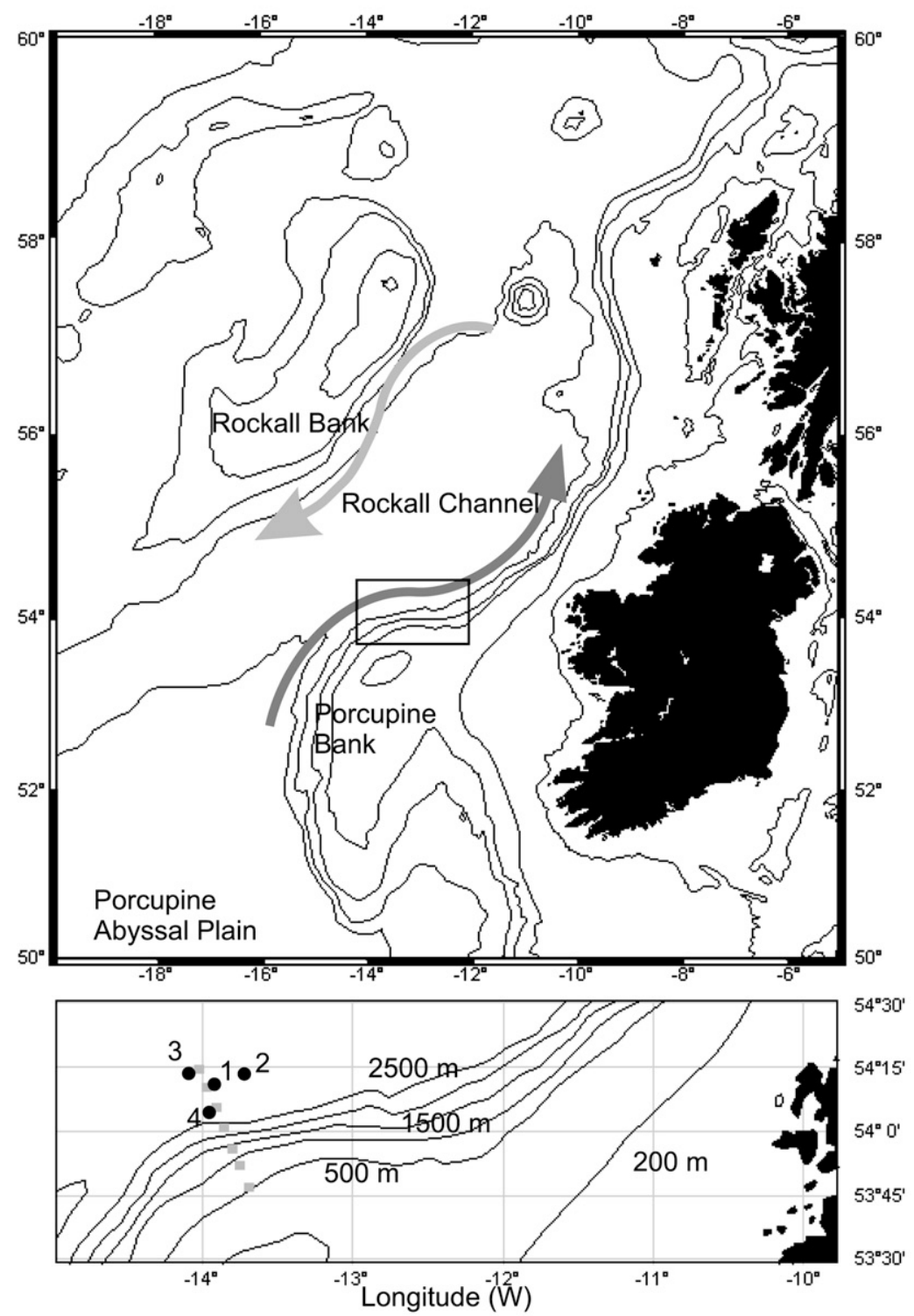



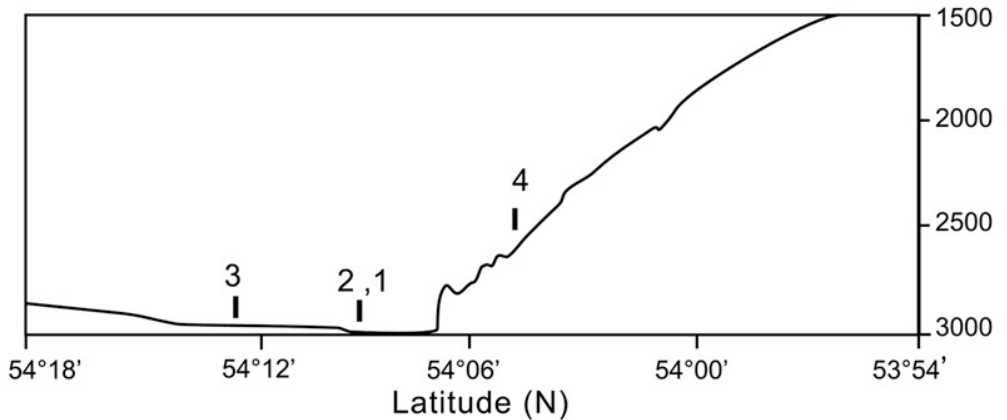

FIG. 2. (top) Map of the area showing the location of the different moorings; data from mooring 1 are analyzed here. The dark gray and bright gray arrows indicate the direction of the mean flow and isobaths are displayed with contour lines 100 and $200 \mathrm{~m}$ and then 500,1500, and $2500 \mathrm{~m}$. (middle) A zoom over the moorings, where each mooring is denoted by a number and isobaths are indicated as well with additional isobaths 1000 and $2000 \mathrm{~m}$ also displayed. (bottom) Vertical section in the neighborhood of the moorings, where the bottom is displayed with a dark line and moorings are denoted by numbers. 


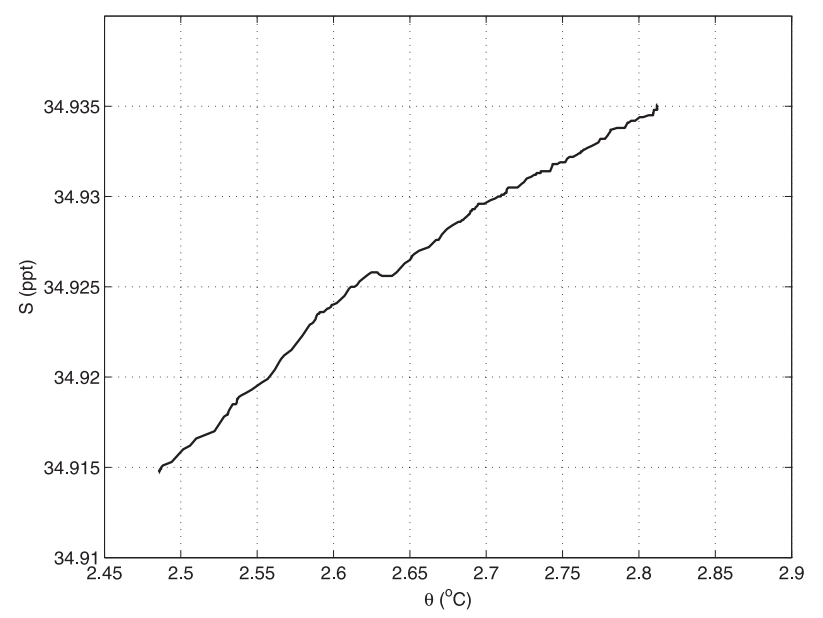

FIG. 3. TS diagram for depths within 2800-2900 m.

\section{Stratified inertial subrange and estimate of kinetic energy dissipation}

Variability at high frequencies is characterized from frequency spectra of temperature variance $\Phi_{T}(\omega)$. A time window of $2 \mathrm{~h}$ is chosen, which ensures that the buoyancy frequency peak is resolved during the IGW and SIE-IGW regimes. Note that with the fast sampling of the temperature sensors, almost 2 decades in the frequency range are resolved beyond $N$, with a frequency cutoff equal to $60 \mathrm{cph}$. Frequency spectra were additionally computed over two inertial periods to determine the energy level within the IGW frequency band.

Examples of both spectra for the regimes, IGW and SIE-IGW, are displayed in Fig. 7. The energy level differs significantly during IGW and SIE-IGW regimes, with values close to that of the GM72 energy level during the first regime (Figs. 7a,b) and higher during the second regime, within a factor of 15 and 30 relative to the GM72 energy level (Figs. 7c,d, respectively).

The shape, energy level, and slope are next inferred from 2-h frequency spectra (Fig. 8a). Note that, because the spectral slope of frequency spectra is the same as that of horizontal wavenumber spectra next inferred using Taylor hypothesis, it is discussed here for convenience. During these two regimes, the shapes of the spectra at super buoyant frequencies, $\omega \in I=[2 N, 30 \mathrm{cph}]$ follow a power law with a mean spectral slope equal to $-1.83 \pm$ 0.06 (Fig. 8a). This slope is close to that of an inertial range, which is equal to $-5 / 3$ with a bias toward a steeper slope. This bias is slightly higher during the SIE-IGW regime with a mean slope value of -1.84 , as compared with the -1.82 mean slope during the IGW regime. A visual inspection of the spectra shows that the slope is slightly steeper in the lower frequency range of $I$ during regimes IGW and SIE-IGW. Indeed, when the mean slope is computed over a restricted frequency range $\omega \in$ $I_{\mathrm{hf}}=[3,30 \mathrm{cph}]$ a value equal to $-1.73 \pm 0.07 \%$ is obtained (Fig. 8a). In contrast, when the stratification is almost neutral, during the regime WSN, the averaged slope computed over $I_{\mathrm{hf}}$ is equal to $-5 / 3$.

This small low bias specific to the IGW and SIE-IGW regimes is a result of the mean stable stratification leading to steeper slopes around $N$, following the typical weak spectral hump just below $N$. To analyze more precisely the shapes of the spectra and the transition between the IGW range and the superbuoyant frequency range, local slopes were computed over subintervals $I_{n}=\left[\omega_{n}, 3 \omega_{n}\right]$ with $\omega_{n} \in[0.5 N, 10 \mathrm{cph}]$ using frequency spectra computed over $2 \mathrm{~h}$. The steepest slopes, equal to -3 , are generally observed within a frequency band $\omega_{n} \in$ $\left[N_{2 \mathrm{iP}}, 2 N_{2 \mathrm{iP}}\right]$, where $N_{2 \mathrm{iP}}$ is the mean buoyancy frequency averaged over two inertial periods. This -3 range extends over higher frequencies during a few short time periods, around days 209, 225, and 237. Two examples are displayed in Figs. 7b,c. The occurrence of these cases of steep slopes for $\omega>2 N$ is very rare as seen on the probability distribution function (PDF) of local slopes computed over $I$ (Fig. 9): beyond the $2 N$ frequency value, the probability of encountering local slopes steeper than -2.76 is on the order of $1.5 \%$.

\section{a. Horizontal wavenumber spectra deduced from a local Taylor hypothesis}

A classical method for inferring horizontal wavenumber spectra from frequency spectra is to use the Taylor hypothesis (e.g., Zaman and Hussain 1981; Pinton and Labbé 1994). In the presence of a large-scale mean flow $U$, temporal measurements $\left[T\left(t_{i}\right) ; t_{1}, t_{2}=t_{1}+\Delta t \ldots\right]$ can be transformed into spatial variations $T\left(t_{i}\right) \sim T\left(x_{i} / U\right)$, provided that the mean flow is relatively large compared to velocity fluctuations and slowly varying with time. This approach has been previously generalized to unsteady flows using a local Taylor hypothesis. A local velocity $U_{l}$ is then defined according to flow conditions. For instance, when the turbulence is forced by a time-varying largescale flow, as in the rotating disk experiments of Pinton and Labbé (1994), $U_{l}$ is defined as the averaged velocity over a period of rotation of the disks. Note that this assumption relies on a physical argument, namely that the period of rotation of the disks corresponds to that of the energy input into turbulence. In a configuration closer to the present case, that of a breaking large-scale internal gravity wave, the local Taylor hypothesis rests upon the assumption that turbulence is primarily fed by the breaking of IGW. A local Taylor velocity is thus defined as the rms of the velocity in the IGW frequency band. This definition was applied to laboratory experiments in which a high-frequency internal gravity wave was forced 

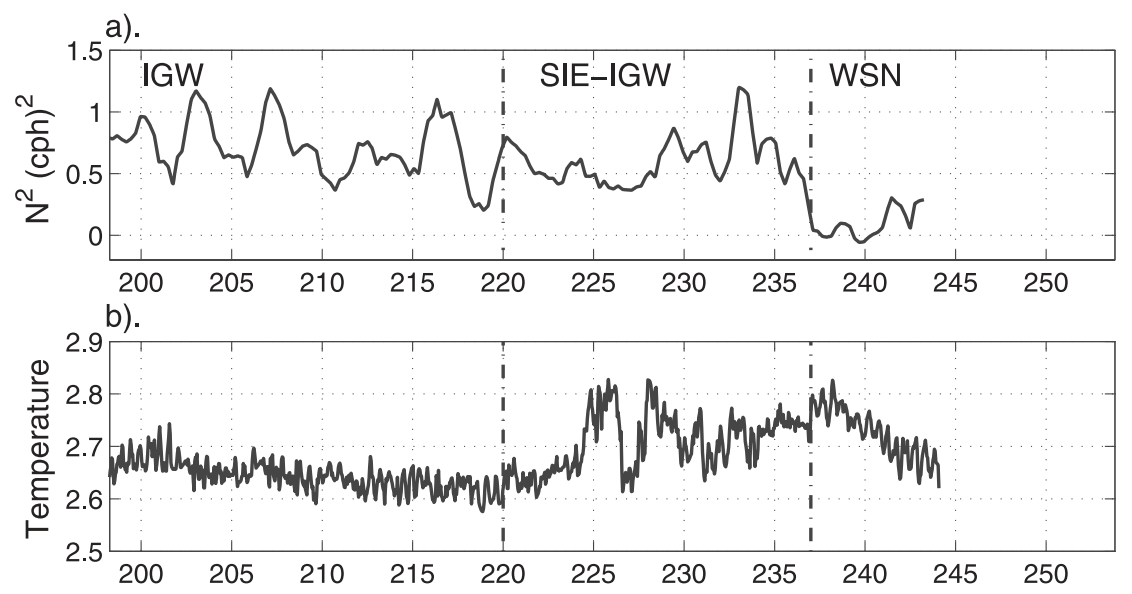

c).

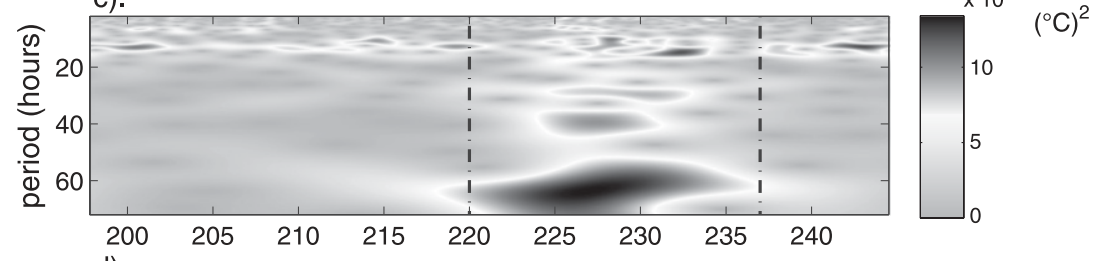

d).


e).

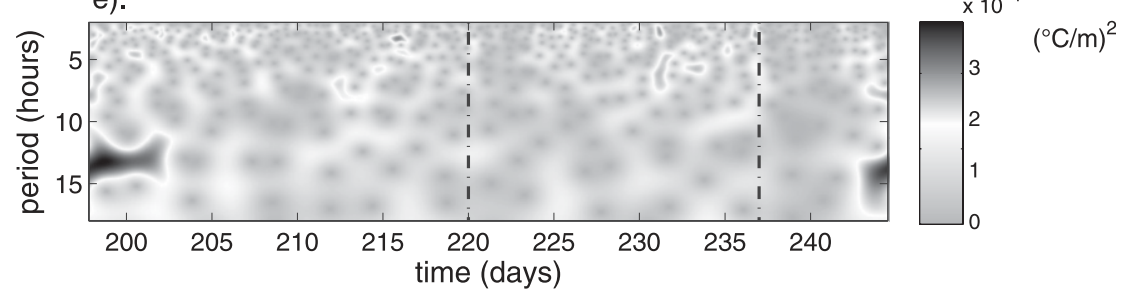

FIG. 4. (a) Buoyancy frequency square averaged over two inertial periods as a function of time with the three regimes, IGW, SIE-IGW and WSN, indicated. (b) Temperature (K) time series at $2893.5 \mathrm{~m}$. (c) Modulus of the wavelet transform of temperature $T$ in the low-frequency range, with periods between 6 and 72 h. (d) As in (c), but in the high-frequency range, with periods between 1 and $18 \mathrm{~h}$. (e) Modulus of the wavelet transform of $\Delta T / \Delta z$ with $\Delta z=16 \mathrm{~m}$, in the high-frequency range.

parametrically by Bénielli and Sommeria (1996). In that case, vertical wavenumber spectra of available potential energy were inferred from frequency spectra, based on the observation that most of the advection was vertical and due to the wave field: $\rho_{t} \sim W \rho_{z}$, where $W$ is the rms of the vertical velocity of the internal wave field. Numerical simulations replicating these laboratory experiments confirmed the validity of the method (Bouruet-Aubertot et al. 1996).

There is a great similarity between the case described above and present observations for which internal tidal motion dominates. However, here the semidiurnal tidal currents are mostly horizontal because the semidiurnal frequency is close to the inertial frequency at this latitude, $M_{2}=1.2 f$. Hence most of the advection is expected to be horizontal with the following balance: $T_{t}^{\prime} \sim U T_{z}^{\prime}$, where $U$ is the horizontal Taylor velocity defined thereafter. In addition, the dynamics is more complex in the observations with significant subinertial motions also present during the SIE-IGW regime. Thus, we define a local Taylor velocity $U$ as the absolute value of the horizontal current averaged over $2 \mathrm{~h}$ (Fig. 10). This 

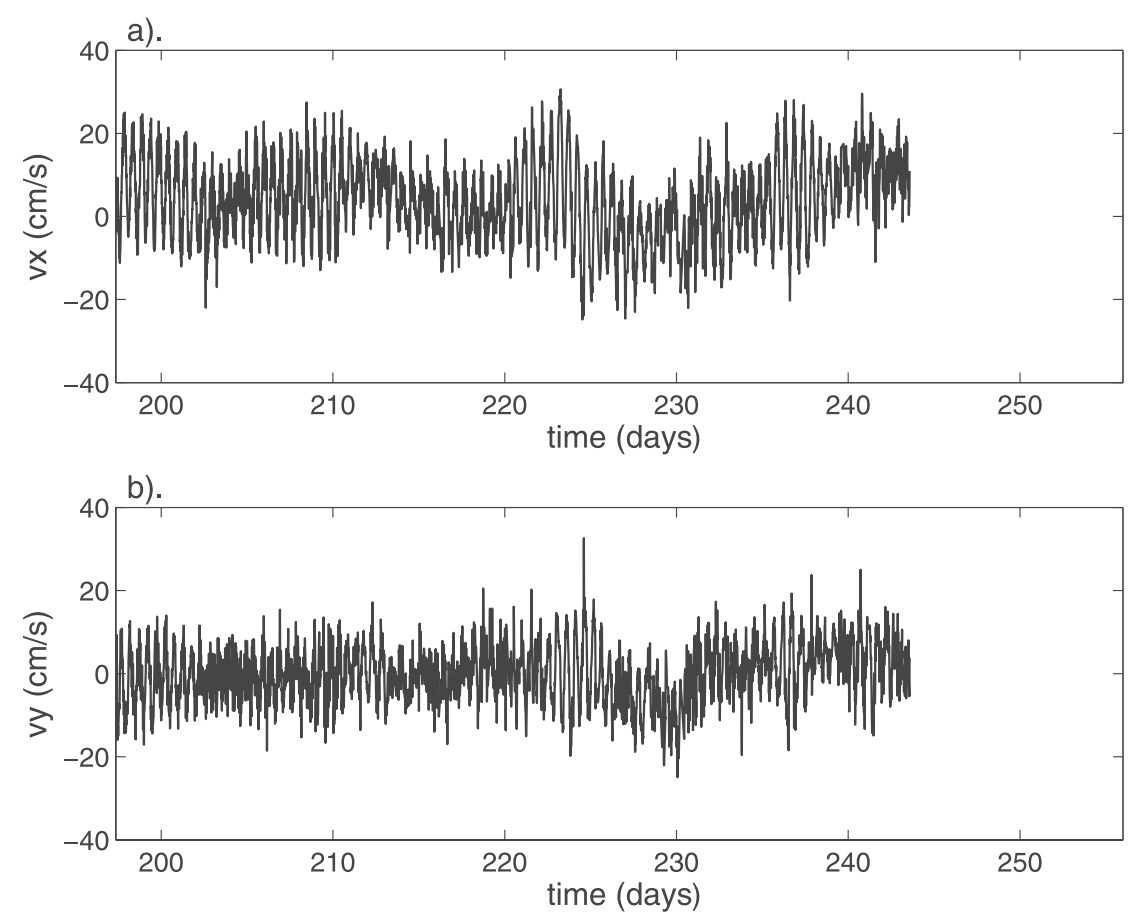

c).

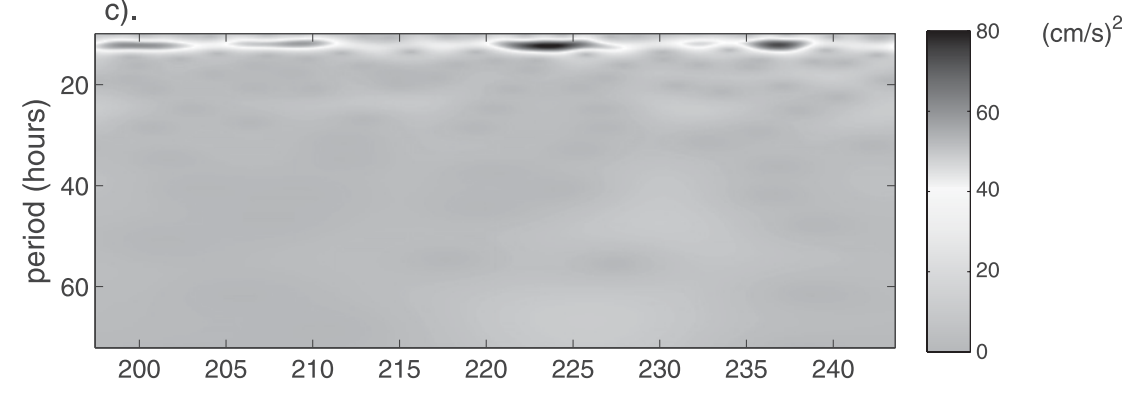

d).

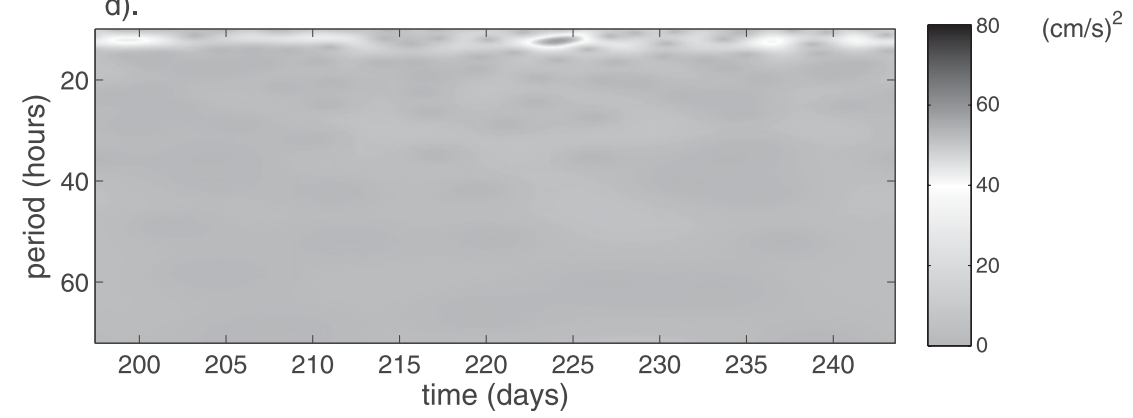

FIG. 5. (a) Zonal and (b) meridional velocity components $\left(\mathrm{cm} \mathrm{s}^{-1}\right)$ at $2920 \mathrm{~m}$ as functions of time. (c) Modulus of the wavelet transform of (c) zonal $\left(v_{x}\right)$ and (d) meridional velocity $\left(v_{y}\right)$ in the low-frequency range, with periods between 10-72 $\mathrm{h}$.

ensures that the internal tidal current does not vary significantly over this time interval and takes into account the subinertial variability. We checked the relevance of this decomposition between velocity fluctuations and a "mean" (i.e., local Taylor velocity) by computing the ratio of their standard deviations. This ratio, displayed in
Fig. 11a, lies within the interval $10^{-2}$ and $10^{-1}$, which validates the decomposition. Second, one needs to check that horizontal advection of temperature by the mean horizontal current is indeed a dominant term at high frequencies, in near balance with the temporal derivative of high-frequency temperature fluctuations. Because 

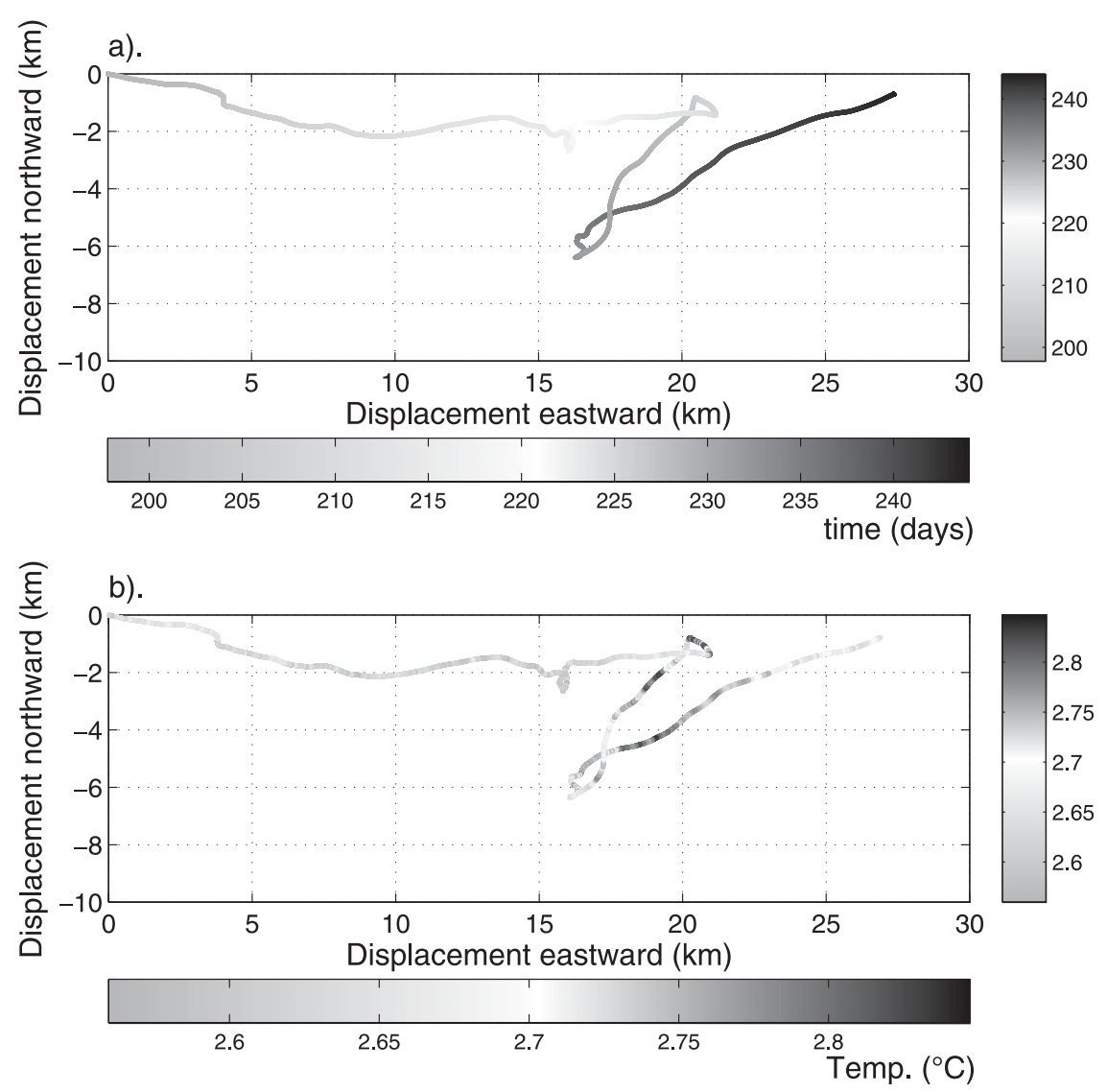

FIG. 6. Progressive vector diagram, where the gray scales refer to (a) time (days) and (b) temperature $\left({ }^{\circ} \mathrm{C}\right)$; the horizontal current has been averaged over two tidal periods for better visualization.

horizontal advection cannot be computed with our dataset, this hypothesis was checked indirectly by comparing vertical advection and temporal derivative of temperature, $T_{t}^{\prime}$ at high frequencies. The estimate of vertical advection of temperature by the mean motion (Fig. 2) $\langle w\rangle T_{z}$ was inferred from high-frequency ADCP measurements $20 \mathrm{~m}$ below the thermistor chain, whereas that of the advection by high-frequency motion $w^{\prime} T_{z}$ was inferred from a synthetic velocity based on the assumption that vertical kinetic energy spectrum follows an inertial subrange at high frequencies. These three terms, $T_{t}^{\prime},\langle w\rangle T_{z}$, and $w^{\prime} T_{z}$, were then filtered using a bandpass filter $(3-30$ $\mathrm{cph})$ to estimate their contribution at superbuoyant frequencies (cf. Fig. 11b). Both vertical advection terms were found to be smaller than the temperature temporal derivative most of the time at these high frequencies. More precisely, we found that vertical advection terms are smaller than $T_{t}^{\prime}$ by a factor of 3 for about $80 \%$ of the values and smaller by a factor of 10 for about $50 \%$ of the values. These estimates suggest that horizontal advection of temperature is mostly performed by the mean flow and that this term must balance most of temporal temperature variations at high frequencies, thus giving confidence in the relevance of the method. The usage of the Taylor hypothesis was further validated with numerical simulations of a breaking internal tide at the Rockall Channel latitude and with similar $N / f$ value of 10 (cf. the appendix).

Horizontal wavenumber spectra of temperature variance $\tilde{\Phi}_{T}\left(k_{h}\right)$ were thus inferred from frequency spectra. Examples characteristic of the different regimes are displayed in Fig. 12. Typical bounds for the horizontal wavenumber $k_{h}$ are from $2 \times 10^{-3}$ to $2 \times 10^{-1} \mathrm{cpm}$, with a $-5 / 3$ domain typically within the $k_{h}$ range from $10^{-2}$ to $10^{-1}$ $\mathrm{cpm}$. The temperature variance spectral level $E_{\mathrm{lev}}$ in the inertial subrange is computed from a log-log regression,

$$
\tilde{\Phi}_{T}\left(k_{h}\right)=E_{\text {lev }}\left(2 \pi k_{h}\right)^{-5 / 3},
$$

and is compared with the theoretical prediction for the inertial subrange. Both the stratified inertial and the inertial convective subranges are of the same form [Tennekes and 

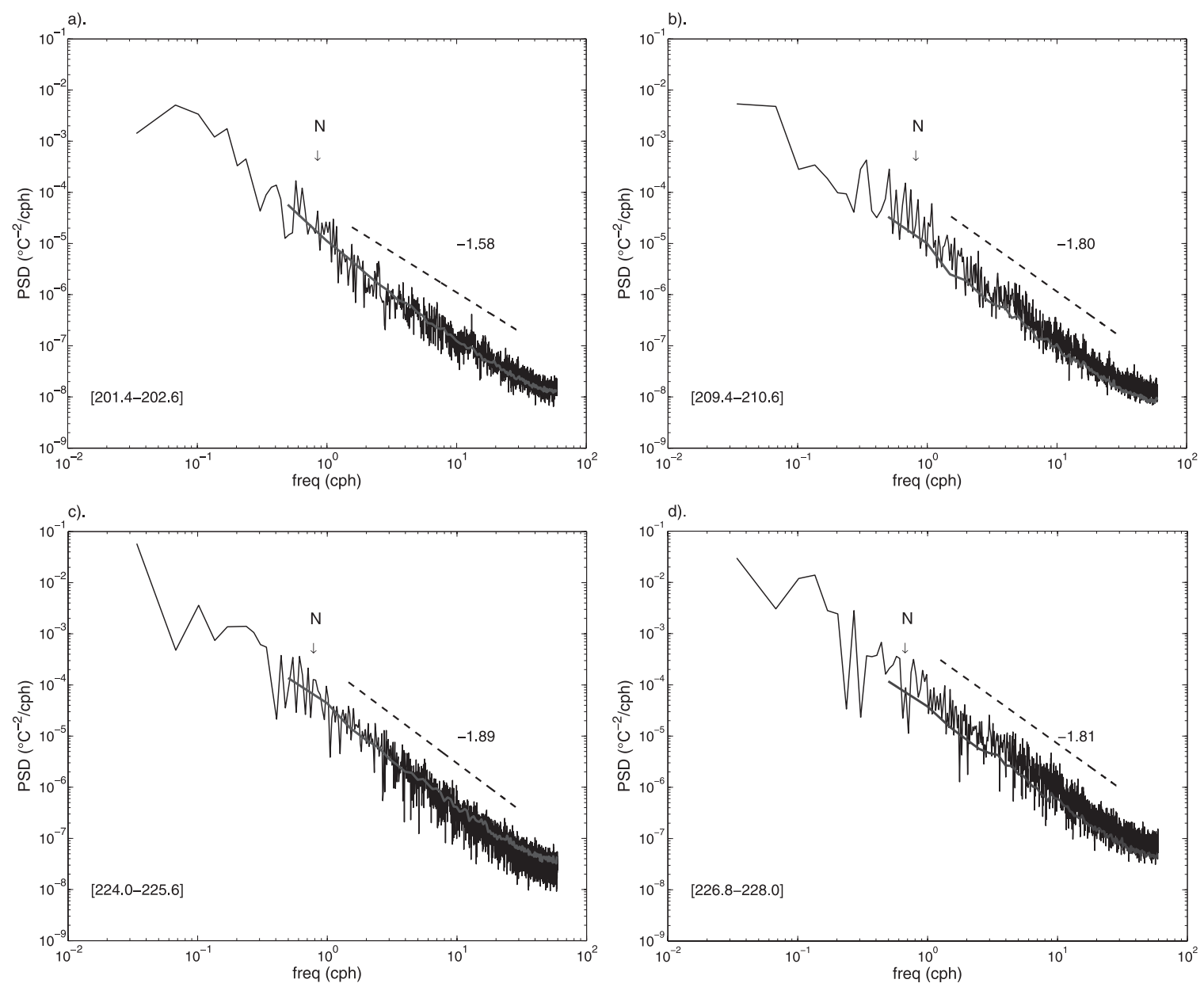

FIG. 7. Frequency spectra of temperature variance averaged over 2893.5-2909.0 m; spectra computed over two inertial periods are displayed in black, whereas the average over the same time interval of spectra computed over $2 \mathrm{~h}$ are displayed in gray: during the IGW regime over the time intervals (a) 201.4-202.6 days and (b) 209.4-210.6 days, the energy within the IGW frequency band is about that of the GM72 model; (c) during the SIE-IGW regime over the time interval 224.0-225.2 days, the energy within the IGW frequency band is about 15 times that of the GM72 model; and (d) during the SIE-IGW regime over the time interval 226.0-227.2 days, the energy within the IGW frequency band is about 30 times that of the GM72 model. The fit of the spectra at superbuoyant frequencies is displayed with a dashed line with a shift of 0.9 for better visualization.

Lumley (1972) and Sreenivasan (1996) for the inertial convective subrange and Riley and Lindborg (2008) for the stratified inertial subrange],

$$
E_{\theta}(k)=C_{\theta} \chi \epsilon^{-1 / 3} k_{h}^{-5 / 3}
$$

where $C_{\theta}$ is a constant (referred to as the ObukhovCorrsin constant for the inertial convective subrange), $\chi$ is the dissipation of temperature variance, and $\epsilon$ is the kinetic energy dissipation. Here, $C_{\theta}$ is set to 0.4 following Sreenivasan (1996). Using the relationship $K_{\rho}=$ $\Gamma_{m} \epsilon / N^{2}$ (Osborn 1980), where $K_{\rho}$ is the eddy diffusivity of density, assuming a constant mixing efficiency $\Gamma_{m}=$ 0.2 and $K_{\rho}=K_{T}=(1 / 2) \chi /\langle d T / d z\rangle^{2}$, where $K_{T}$ is the eddy diffusivity of temperature, this leads to

$$
E_{\theta}\left(k_{h}\right)=2 C_{\theta} \Gamma_{m}[N /(g \alpha)]^{2} \epsilon^{2 / 3} k_{h}^{-5 / 3}
$$

which gives

$$
\epsilon=\left\{\frac{E_{\mathrm{lev}}}{2 C_{\theta} \Gamma_{m}[N /(g \alpha)]^{2}}\right\}^{3 / 2},
$$

and $\epsilon$ and $K_{\rho}$ are displayed in Fig. 13 as well as the dimensionless parameter $I=\epsilon /\left(v N^{2}\right)$, which can be interpreted as the ratio of the buoyancy time scale $1 / N$ to the characteristic time scale for turbulent events to fully develop $(\epsilon / v)^{1 / 2}$ (Ivey et al. 2008). Values for $\epsilon$ lie within the range $1 \times 10^{-9}$ and $3 \times 10^{-7} \mathrm{~m}^{2} \mathrm{~s}^{-3}$. These values are consistent with the wide range of variation for $\epsilon$ inferred 

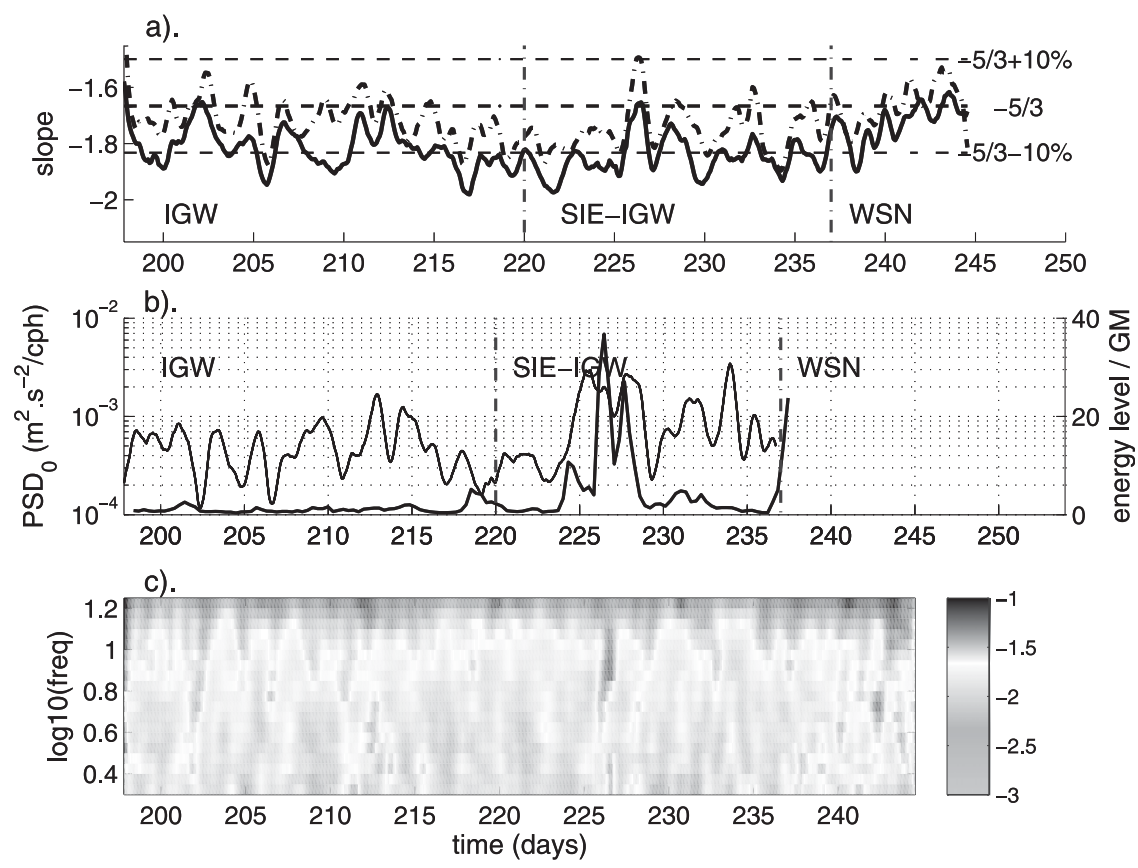

FIG. 8. (a) Slope of temperature variance spectra for $\omega>N\left\{\omega \in\left[\omega_{0}, 1 /(2 d t)\right]\right\}$ as a function of time; the black solid line is for $\omega_{0}=2 N$ and the black dotted line is for $\omega_{0}=6 \mathrm{cph}$, and the slopes $-5 / 3$ and $-5 / 3 \pm 10 \%$ are indicated by three dashed straight lines. (b) Power spectral density of temperature variance spectra at the beginning of the inertial subrange $\omega=2 \mathrm{~N}$ : $\mathrm{PSD}_{0}$ (black curve); the ratio between IGW energy level and GM72 level is also represented (gray curve). (c) Local slope computed over a frequency interval $\left[\omega_{n}, 3 \omega_{n}\right]$ as a function of time and frequency.

from observations, typically within $10^{-9}$ and $10^{-5} \mathrm{~m}^{2} \mathrm{~s}^{-3}$. Dimensionless parameter $I$ is high, with values larger than 500, averaging 24000 , thus far beyond the upper limit for intermediate regimes, which is equal to 100 . The time evolution of $I$ shows that smaller values are obtained during the IGW regime in contrast with higher values within the time interval (225-229 days) during the SIEIGW regime, consistent with higher IGW energy levels during this time interval. Estimated values for $K_{\rho}$ are high, within $2 \times 10^{-5}$ and $3 \times 10^{-3} \mathrm{~m}^{2} \mathrm{~s}^{-1}$, partially as a result of the weak stratification. However, there is an uncertainty in the estimate of $K_{\rho}$ resulting from the applicability of the Osborn relationship for weak stratification, as well as on the estimate of $N$ because of the absence of continuous salinity measurements.

The Ozmidov scale $\lambda_{O}=\left(\epsilon / N^{3}\right)^{1 / 2}$ is next inferred (Fig. 14). Large $\lambda_{O}$ values are obtained within [0.4 m, $22 \mathrm{~m}$ ] until day 235, which corresponds to Ozmidov wavenumbers between $4 \times 10^{-2}$ and $2.5 \mathrm{cpm}$. The Ozmidov wavenumber thus sometimes falls within the range where a spectral slope of $-5 / 3$ is obtained. Hence, a few horizontal wavenumber spectra give evidence of the transition between stratified inertial subrange and inertial convective subrange (e.g., Figs. 12b,c).

\section{b. Scaling for energy dissipation}

We seek for a scaling of $\epsilon$ as a function of the energy level in the IGW range (Fig. 15). To this aim, we perform a shifting average of $\epsilon$ over two inertial periods and plot it against potential energy in the IGW frequency range

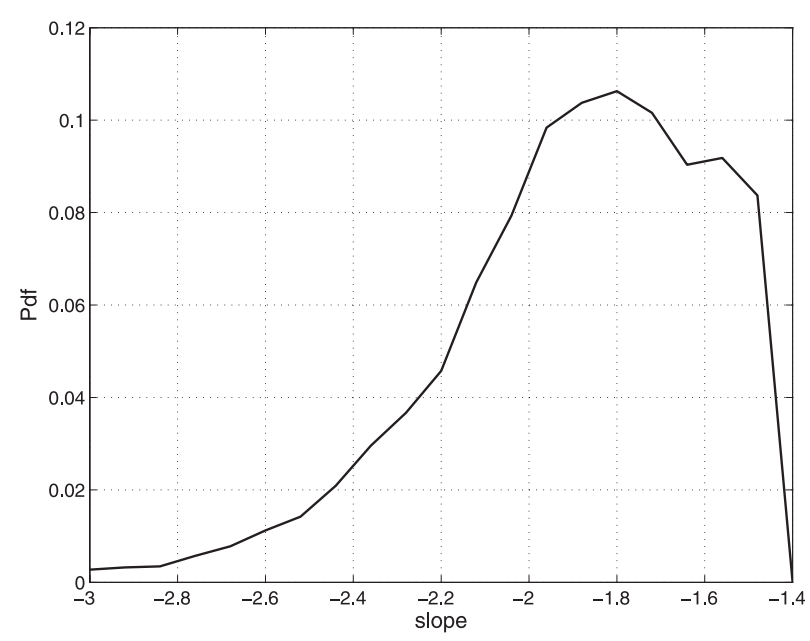

FIG. 9. PDF of the local slopes computed over subintervals $\omega \in\left[\omega_{1}, 3 \omega_{1}\right]$ with $\omega_{1} \in[2 N, 10 \mathrm{cph}]$. 


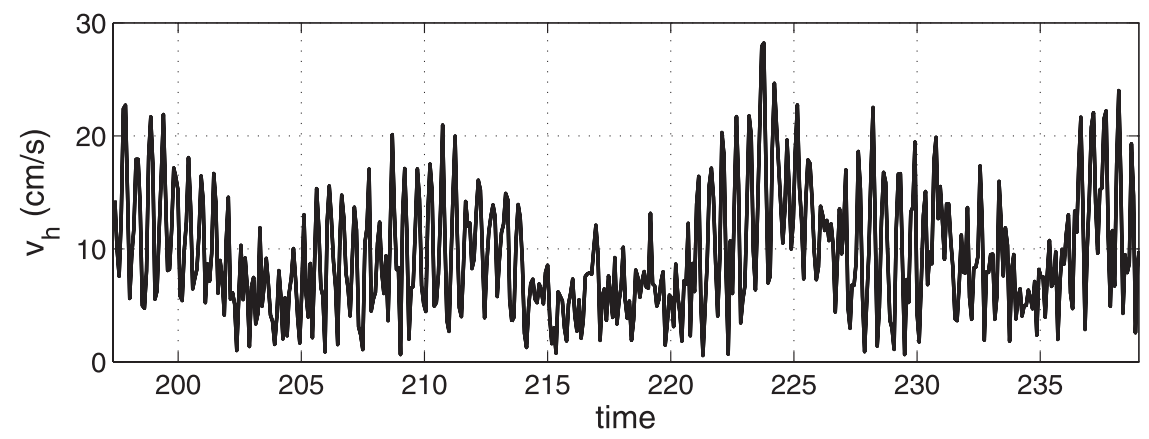

FIG. 10. Modulus of the horizontal velocity averaged over a 2-h shifting window as a function of time.

$\mathrm{PE}_{\mathrm{IGW}}$, inferred from spectra computed over two inertial periods divided by a typical time scale, namely $1 / N$, for dimensional consistency. A $\log -\log$ linear regression of $\epsilon$ as a function of $\mathrm{PE}_{\mathrm{IGW}} N$ is performed. A slightly higher slope is obtained during the IGW regime than during the SIE-IGW regime with the following expressions:

$$
\epsilon=7 \times 10^{3}\left(\mathrm{PE}_{\mathrm{IGW}} N\right)^{3 / 2}
$$

for the IGW regime and

$$
\epsilon=0.24 \mathrm{PE}_{\mathrm{IGW}} N
$$

for the SIE-IGW regime.

Prior to analyzing the vertical temperature structure, the Ozmidov scale is compared with the $0.5-\mathrm{m}$ vertical sampling to get insight into the dynamical range [stratified turbulence (ST), inertial convective subrange (ICS)] that is resolved (Fig. 14). The Ozmidov scale is generally larger than the $0.5-\mathrm{m}$ vertical separation between temperature sensors, except around days 216 and 221 (Fig. 14).
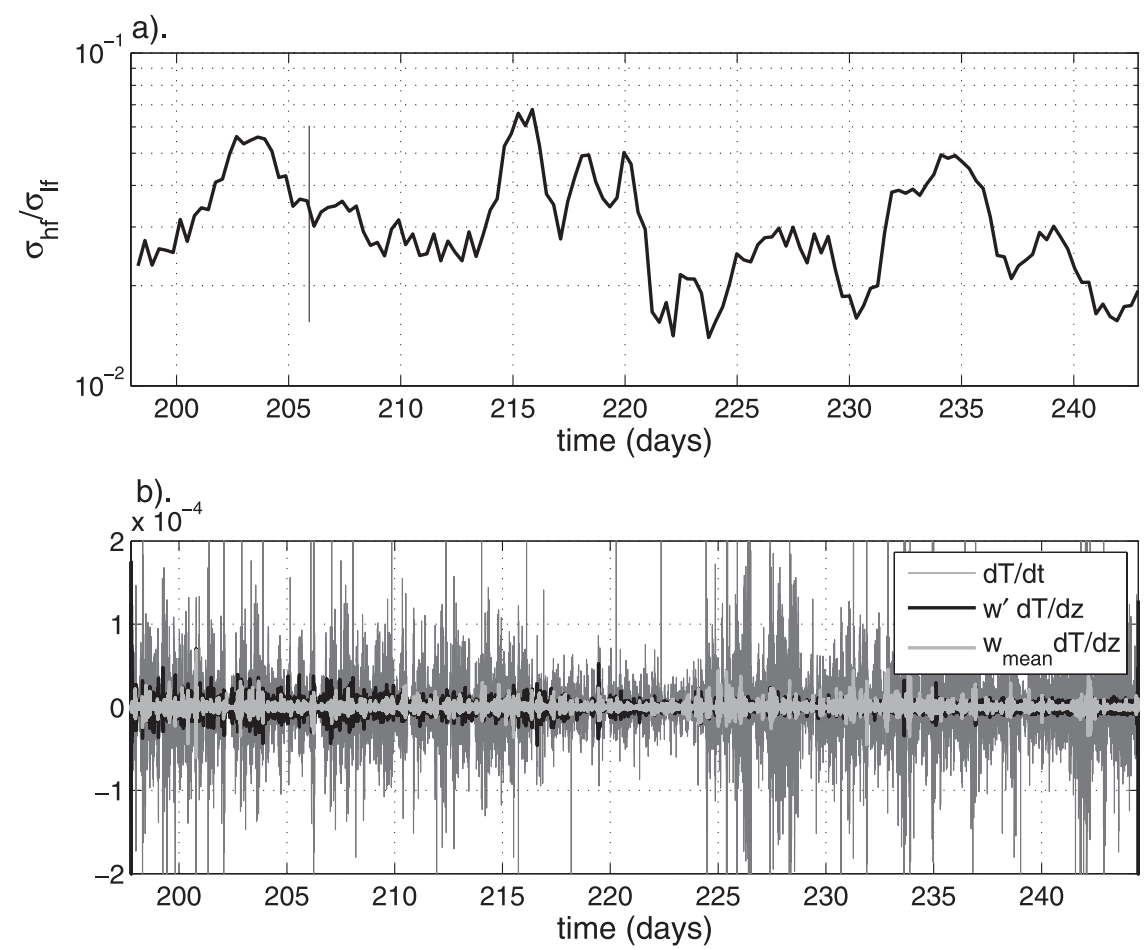

FIG. 11. (a) Ratio between standard deviation of high-frequency horizontal current $(\omega>$ $0.5 \mathrm{cph}) \sigma_{\mathrm{hf}}$ and low-frequency horizontal current $(\omega>0.5 \mathrm{cph}) \sigma_{\mathrm{lf}}$ as a function of time. (b) Time series of temporal derivative of high-frequency temperature fluctuations (dark-gray curve) and vertical advection of temperature fluctuations by low-frequency motion (light-gray curve) and by high-frequency motion (black curve). 

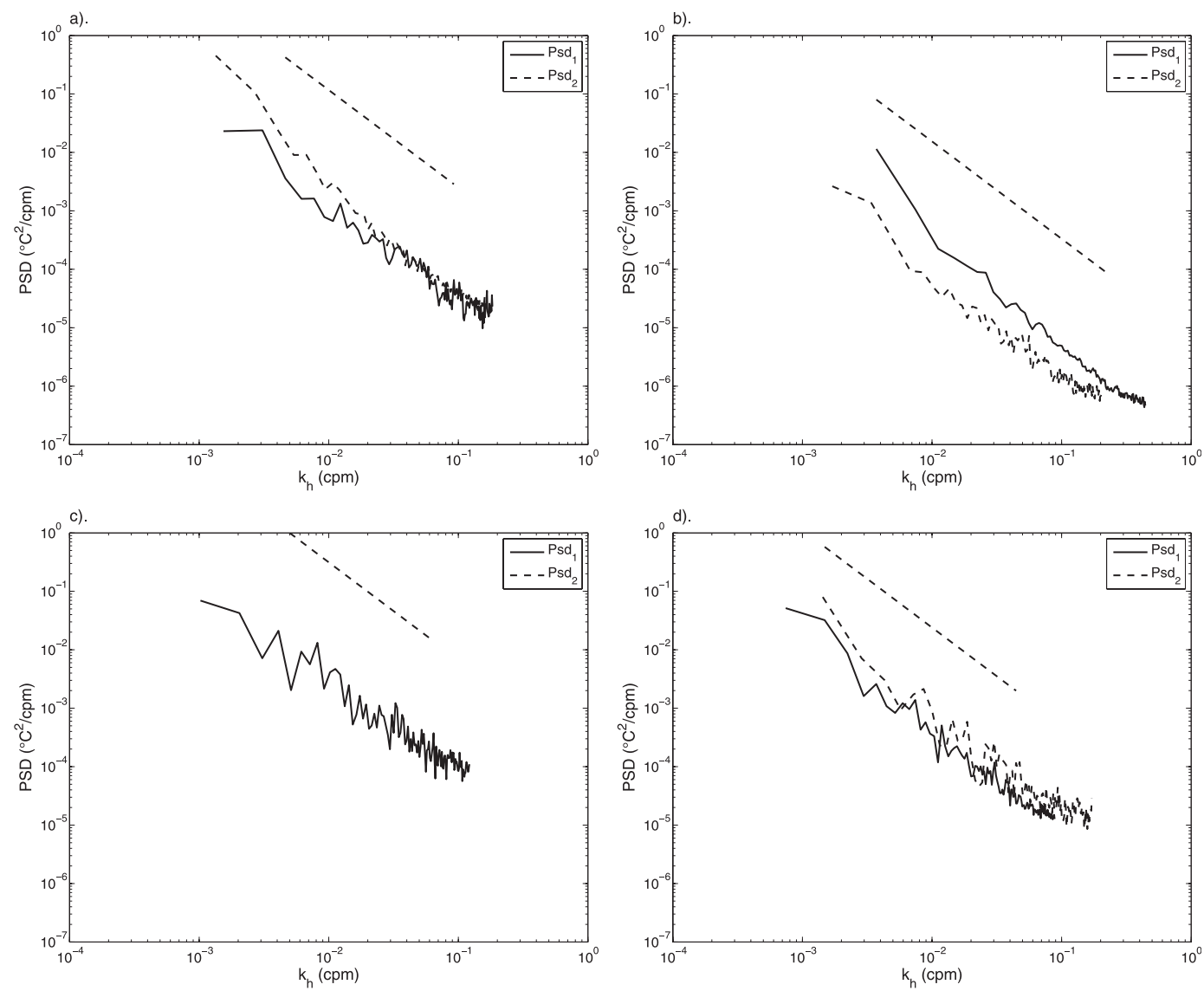

FIG. 12. Horizontal wavenumber temperature variance spectra inferred from frequency spectra computed over a 2-h time window using a local Taylor hypothesis; a few spectra, referred to as $\mathrm{Psd}_{1}$ and $\mathrm{Psd}_{2}$, are displayed during the IGW regime around days (a) 202 and (b) 218 and during the SIE-IGW regime around days (c) 226 and (d) 227; the -5/3 slope is shown for comparison. The Ozmidov wavenumber $k_{O}$ is equal to (a) $0.23 \mathrm{cpm}$ for $\mathrm{Psd}_{1}$ and $0.26 \mathrm{cpm}$ for $\mathrm{Psd}_{2}$; (b) $0.046 \mathrm{cpm}$ for $\mathrm{Psd}_{1}$ and $0.047 \mathrm{cpm}$ for $\mathrm{Psd}_{2}$; (c) $0.054 \mathrm{cpm}$ for Psd $\mathrm{Pd}_{1}$ and (d) $0.10 \mathrm{cpm}$ for Psd $\mathrm{Pand}_{1} 0.059 \mathrm{cpm}$ for Psd 2 .

\section{Discussion}

The analysis of temperature variance spectra reveals a transition to an inertial range at a frequency of about twice the buoyancy frequency, for various dynamical regimes of high or low energy levels. Very few observations report on this superbuoyant frequency range using Eulerian measurements in the ocean (e.g., McPhaden and Peters 1992; Lien et al. 1996), and the spectral shape has not previously been detailed. Most frequency spectra are inferred from Lagrangian floats and exhibit the classical -2 slope (D'Asaro and Lien 2000a). Seuront and Schmitt (2004) gave evidence of these two regimes, Lagrangian and Eulerian, using temperature measurements in an almost nonstratified environment, the English Channel. A -2 slope, typical for Lagrangian turbulence, was obtained for large time scales for which the motion of the ship was negligible, in contrast to the $-5 / 3$ slope, typical for Eulerian turbulence, which was observed at small time scales. This $-5 / 3$ slope is retrieved here, but with a negative bias of $9 \%$ toward steeper slopes. This bias, which is observed in the presence of a mean stable stratification, results from the intermittency related to the occurrence of strongly stratified sheets (Bouruet-Aubertot et al. 2004). It is consistent with theoretical models for intermittency, which predict a steeper slope (e.g., Frisch 1995). For instance, the $\beta$ model predicts a $-\left(5 / 3+\mu^{\prime} / 3\right)$ slope with $\mu^{\prime}=0.2$. This bias, $\sim 4 \%$, is slightly smaller than the $9 \%$ bias observed here, which suggests that intermittency is increased in the presence of mean stable stratification. This point could be addressed with a larger set of data than available here.

A local Taylor hypothesis is used to infer horizontal wavenumber spectra. Because the motion is dominated by semidiurnal tides and is nearly horizontal, the advective velocity is taken as the absolute value of the horizontal current averaged over $2 \mathrm{~h}$. This method has been validated recently in numerical simulations of breaking IGW (see appendix; P. Bouruet-Aubertot and M. P. Lelong 2010, unpublished manuscript). The inertial 

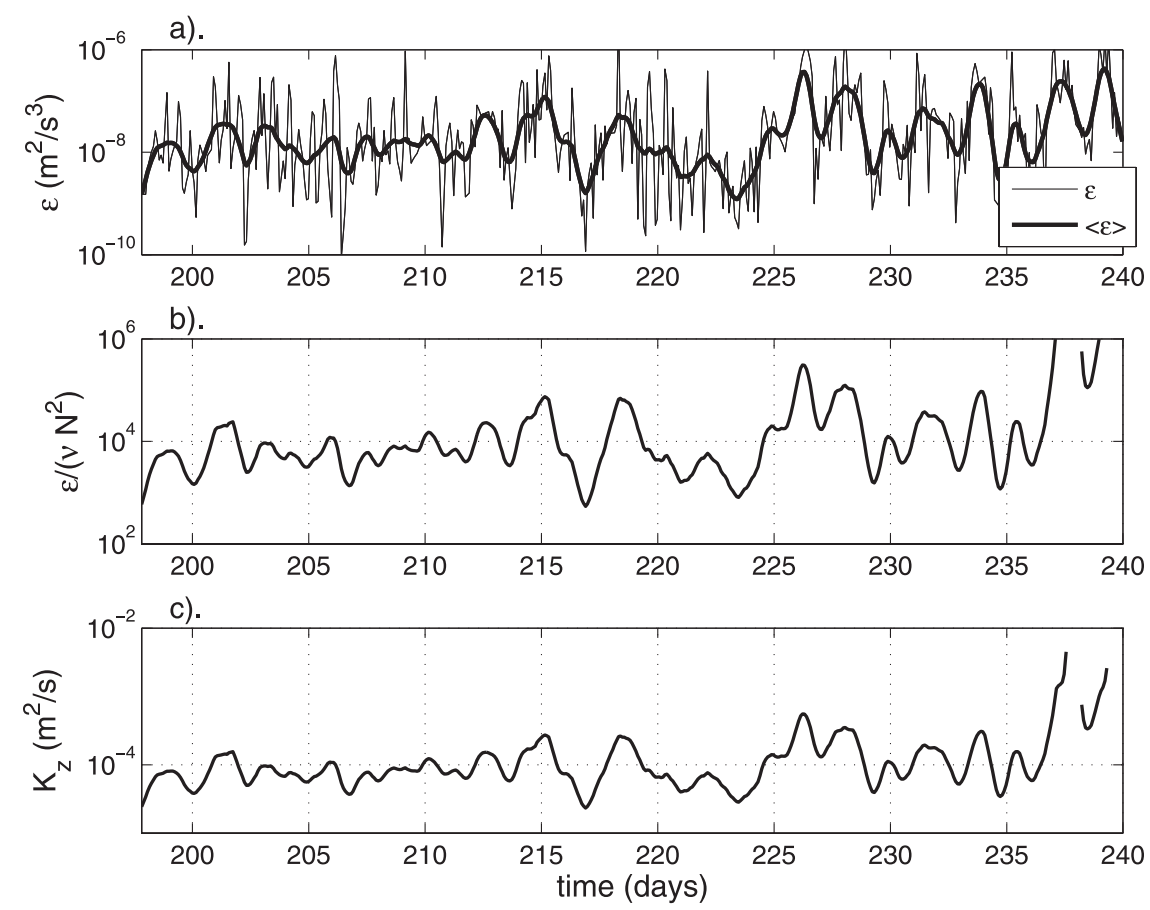

FIG. 13. (a) Kinetic energy dissipation rate $\epsilon$ inferred from temperature variance spectra as a function of time, where the shifting average of $\epsilon$ over two inertial periods is displayed with a thick line. (b) Shifting average of $I=\epsilon /\left(v N^{2}\right)$ as a function of time. (c) Shifting average over two inertial periods of vertical eddy diffusivity as a function of time.

subrange extends over horizontal wavenumbers within $2 \times 10^{-3}$ and $2 \times 10^{-1} \mathrm{cpm}$. The upper bound of this interval is sometimes higher than the Ozmidov wavenumber, which is typically within $4 \times 10^{-2}$ and $2.5 \mathrm{cpm}$.

Hence, the transition between stratified inertial subrange and convective inertial subrange is sometimes resolved, though in most cases the stratified inertial subrange only is resolved. Interestingly, this interpretation follows that of Riley and Lindborg (2008). Their analysis is based on a set of spectra inferred from observations in the ocean (Holbrook and Fer 2005; Klymak and Moum 2007b) and in the atmosphere (Frehlich et al. 2008) as well as from numerical simulations. Two subdomains are distinguished within the $-5 / 3$ subrange. The first one corresponds to wavenumbers smaller than the Ozmidov wavenumber and is strongly anisotropic with horizontal and vertical wavenumber spectra of different shapes. This results from stratification effects that are dominant, leading to low horizontal Froude number and vertical Froude number of order 1 . Note that the direction of the energy transfers could not have been determined from the data. However, the main source of energy input, from the semidiurnal tide, takes place at large scales, which suggests that internal tide breaking is one main source of turbulence implying a downward energy cascade. In contrast, for the second subrange that corresponds to wavenumbers higher than the Ozmidov wavenumber, the isotropy of the inertial convective subrange is retrieved. These two subranges have the same scaling and are characterized by an energy transfer toward small scales $\epsilon$. Our measurements resolve most of the time the stratified inertial turbulence subrange only. This stratified inertial subrange starts at fairly low wavenumbers on the order of $2 \times 10^{-3} \mathrm{cpm}$, which is comparable to the values obtained by Klymak and Moum (2007a,b) using towed measurements.

Kinetic energy dissipation is found to be proportional to the energy within the IGW range and the buoyancy frequency $\epsilon \simeq 0.24 \mathrm{PE}_{\mathrm{IGW}} N$ during the SIE-IGW regime, whereas a scaling in $\left(\mathrm{PE}_{\mathrm{IGW}} N\right)^{3 / 2}$ is obtained during the IGW regime. The former parameterization is comparable to the parameterization mentioned by D'Asaro and Lien (2000b) for high energy levels that relates $\epsilon$ to the vertical

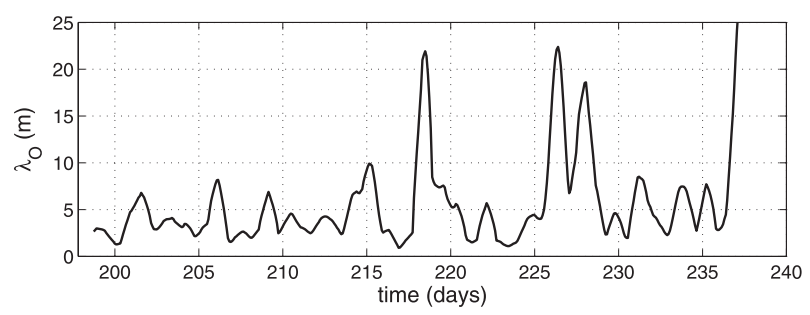

FIG. 14. Ozmidov scale as a function of time. 


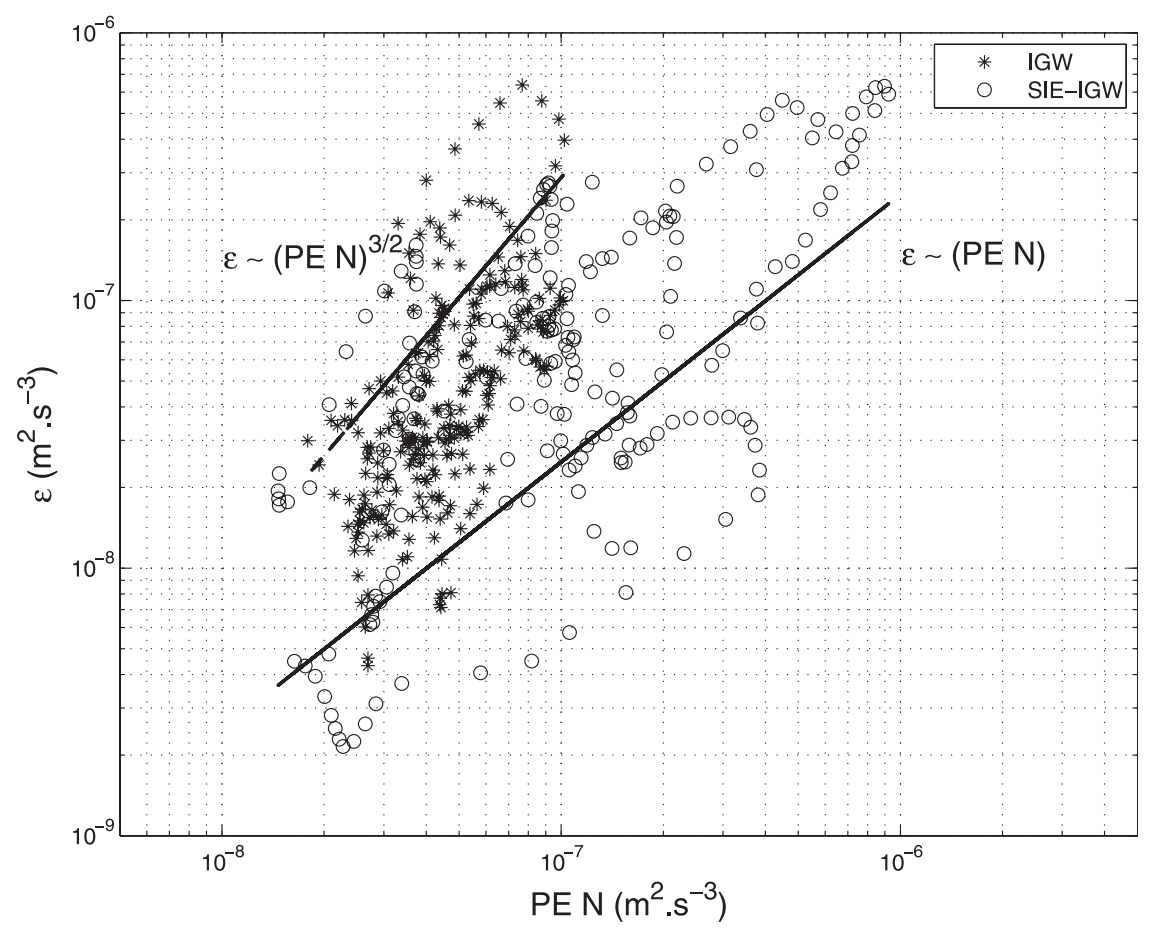

FIG. 15. Kinetic energy dissipation $\epsilon$ as a function of the energy content in the $[f, N]$ frequency band; Points corresponding to the IGW regime are displayed with asterisks and points corresponding to the SIE-IGW regime are displayed with circles. Two fits are displayed: $\epsilon \sim\left(\mathrm{PE}_{\mathrm{IGW}} N\right)^{3 / 2}$ for the IGW regime and $\epsilon \sim \mathrm{PE}_{\mathrm{IGW}} N$ for the SIE-IGW regime.

velocity variance $\sigma_{w}$ times the buoyancy frequency: $\epsilon \simeq$ $C_{0} \beta^{-1} \sigma_{w}^{2} N$, with $C_{0}=0.6$ and $\beta=1.8$, which is equivalent to $\epsilon \simeq 1.9 \mathrm{PE}_{\mathrm{IGW}} N$, using polarization relations. In contrast, the latter parameterization lies in between the $\mathrm{PE}_{\mathrm{IGW}} N$ scaling and the $\left(\mathrm{PE}_{\mathrm{IGW}} N\right)^{2}$ predicted by finescale parameterization for energy levels comparable to Garrett-Munk spectrum. This is consistent with the fact that turbulence level is high in our observations with $\epsilon /\left(v \nu N^{2}\right)$ within the range $5 \times 10^{2}$ and $8 \times 10^{5}$. Similarly, analysis of in situ measurements near topography showed that finescale parameterizations underestimate dissipation above the bottom where burst of turbulence with values of up to $10^{-6} \mathrm{~W} \mathrm{~kg}^{-1}$ were observed (Klymak et al. 2008). Also, the dependence of dissipation as a function of shear was weaker than that predicted by finescale parameterization with a smaller exponent for the power law (Carter and Gregg 2002). All these results, including ours, show that, in regions above topographic features where internal tides are strongly nonlinear, finescale parameterization with a smaller power law exponent should be applied. However, these scalings are not easy to compare, because they are based on different quantities (shear or potential energy).

Note that our estimate of $\epsilon$ from the energy level of the stratified inertial subrange could not have been validated against microstructure measurements, but its relevance has been demonstrated recently by Klymak and Moum (2007a,b), who performed temperature measurements with a towed vehicle. Lien and D'Asaro (2006) also validated the estimate of $\epsilon$ using acoustic Doppler velocimeter measurements and a variant of the Taylor hypothesis.

The estimate of $\epsilon$ from mooring measurements at high frequencies using a generalized Taylor hypothesis based on the mean horizontal advection velocity constitutes, when applicable, a useful tool for increasing the number of estimates of $\epsilon$. It allows a relationship to be established between energy dissipation and lower-frequency motions: for instance, IGW or subinertial motions over long time series. Thus, parameterizations that relate energy dissipation to the instability process responsible for the onset of turbulence and/or the IGW field could be inferred. A finescale parameterization was obtained here in the bottom boundary layer, in a region of high energy level. The method is of particular interest in this region, the bottom boundary layer, where microstructure profiles are rare.

Further measurements of both velocity and temperature are required to investigate jointly potential and kinetic energy spectra, to gain further insight into the instability mechanisms for different dynamical regimes, and to establish and validate parameterizations. Also, numerical simulations of breaking IGW could play a role in 
(a)

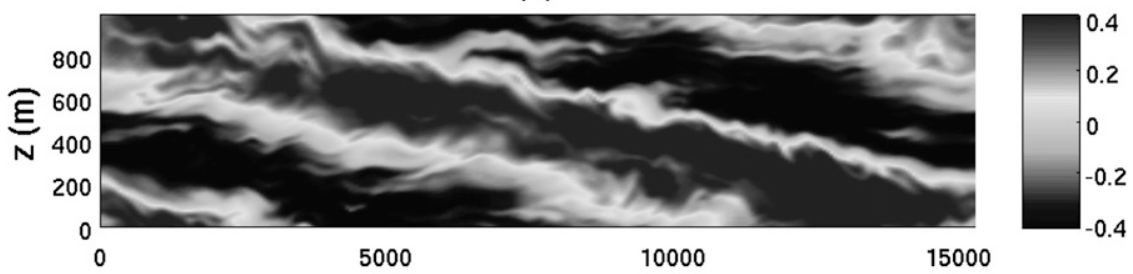

(b)

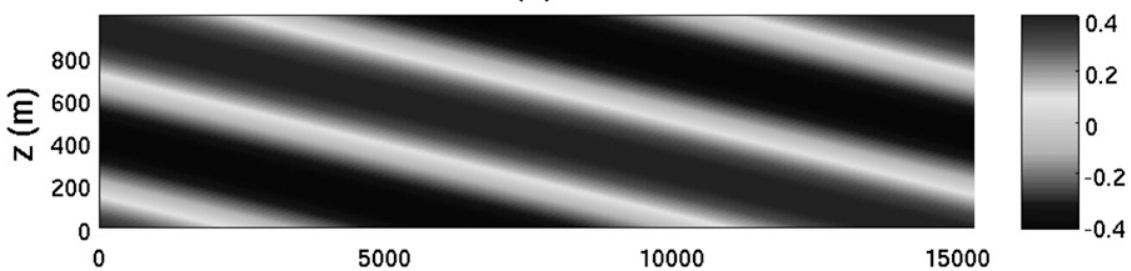

(c)

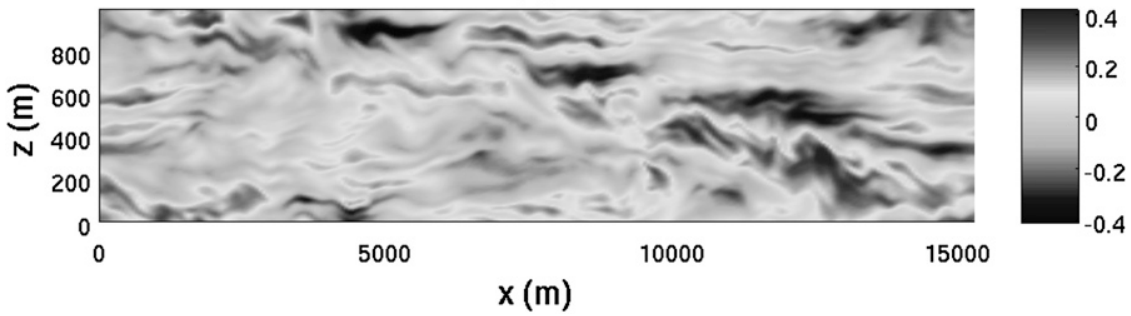

FIG. A1. Vertical section of the eastward component of the velocity $\left(\mathrm{m} \mathrm{s}^{-1}\right)$ : (a) total velocity, (b) velocity of the wave, and (c) perturbation velocity.

investigations involving joint analysis of Eulerian and Lagrangian data.

Acknowledgments. We acknowledge J. Riley for enlightening discussion on stratified turbulence; the reviewers and the editor, E. Kunze, for their comments; and G. Reverdin and Y. Cuypers for useful discussion. The Netherlands Organization for the Advancement of Scientific Research (NWO) is acknowledged for the financial support for the collaboration. This work has been supported by LEFE/IDAO from the Institut National des Sciences de l'Univers.

\section{APPENDIX}

\section{On the Use of Taylor Hypothesis}

Numerical simulations were performed to verify the validity of the generalized Taylor hypothesis for a turbulence evolving in a background flow dominated by a semidiurnal tide. The simulation presented here was set up to model the temporal evolution of a breaking IGW of semidiurnal frequency at the latitude of the mooring site. Other parameters such as stratification and tidal amplitude were chosen to match the observations.

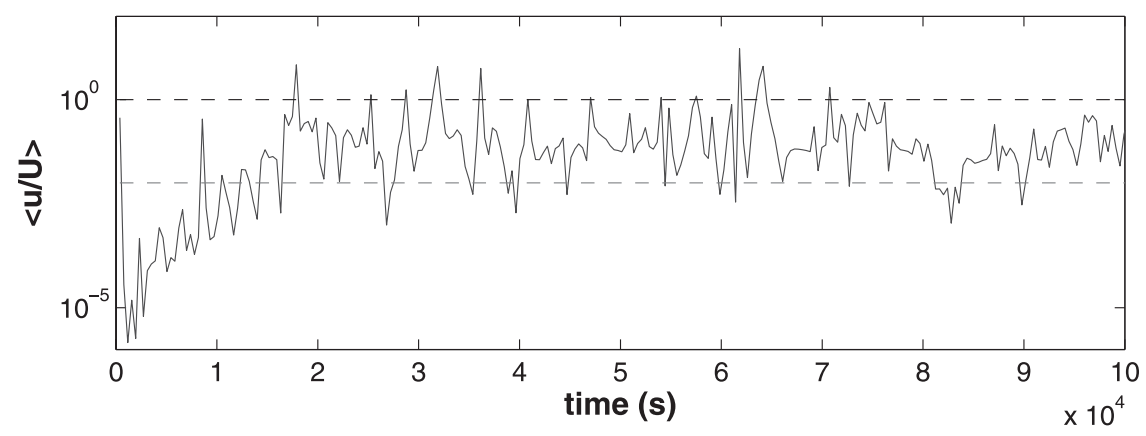

FIG. A2. Volume-averaged ratio of the $x$ component of velocity fluctuation to tidal $x$ velocity as a function of time. Dotted lines indicate values of 0.01 and 1 . 


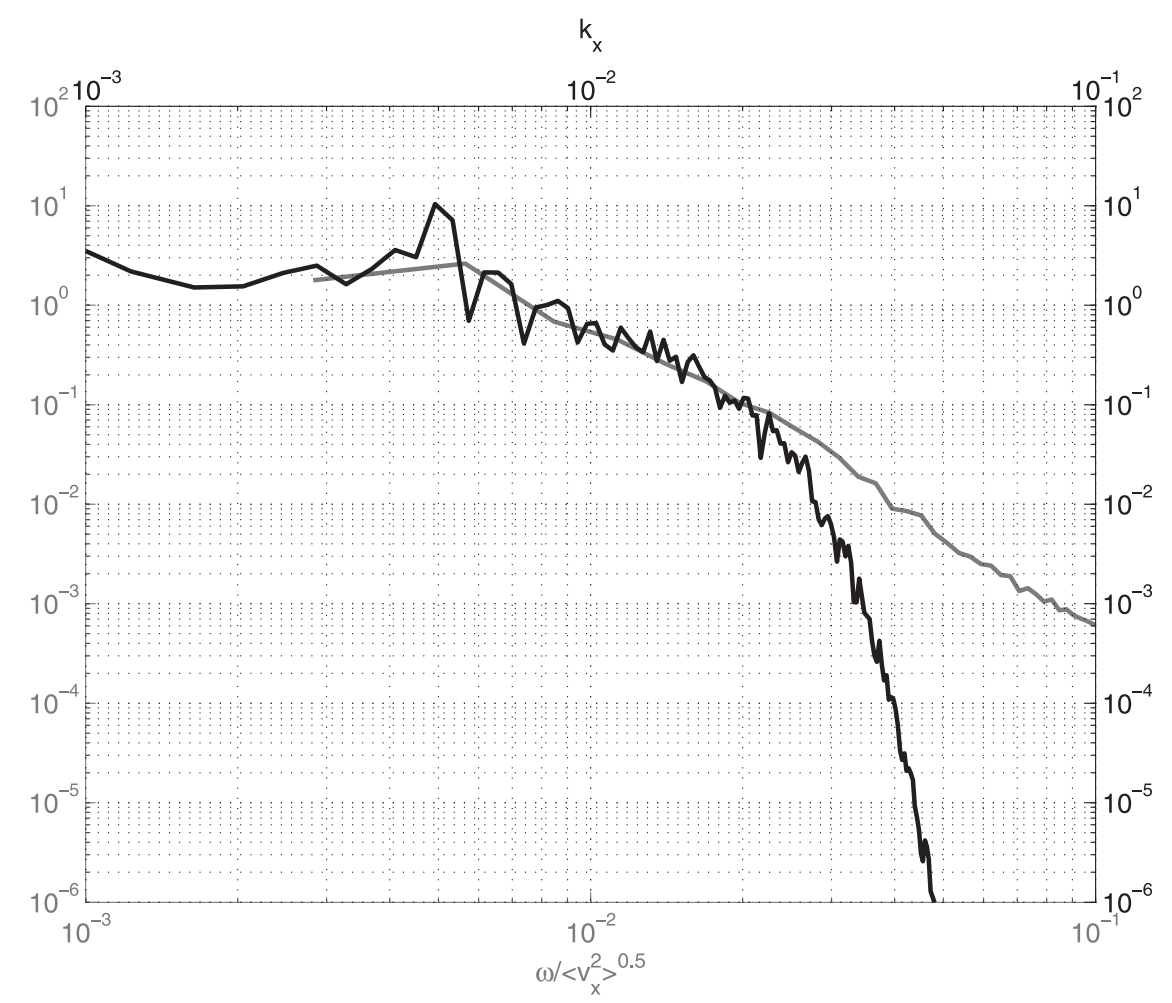

FIG. A3. Horizontal wavenumber spectra of temperature variance at one numerical mooring; the spectrum inferred from frequency spectrum computed over the last two buoyancy periods using a local Taylor hypothesis based on the averaged horizontal velocity is the less jagged line and the average over the same time interval of horizontal wavenumber spectra computed directly is the more jagged line. Superposition of the two spectra is obtained within the wavenumber range $9 \times 10^{-3}$ and $2 \times 10^{-2} \mathrm{cpm}$ in the stratified inertial subrange with a slope close to $-5 / 3$.

The numerical setup is similar to that used by Lelong and Dunkerton (1998a,b) in their study of IGW instabilities. The initial condition consists of randomly phased white noise superimposed on a single, linear inertiagravity wave with rightward and upward phase propagation, having dimensional wavenumber $(k, l, m)=\left(k_{\min }\right.$, $\left.0, m_{\min }\right)$ in a computational domain of dimension $L_{x}=$ $L_{y}=2 \pi / k_{\min }$ and $L_{z}=2 \pi / m_{\min }$. We have chosen, without loss of generality, to orient the coordinate system such that the wave propagates is in the $x-z$ plane. The viscous operator consists of a combination of Laplacian viscosity and sixth-order hyperviscosity in vertical and horizontal directions, respectively.

To verify whether the Taylor hypothesis can be used, we must demonstrate that the mean velocity at a fixed point is much greater than the turbulent velocities, implying that advection due to turbulent motions is small compared to that by the mean flow. At the Rockall Channel latitude, semidiurnal and inertial frequencies are very close and the tidal flow is nearly circularly polarized. Consequently, it is sufficient to examine the behavior of one of the horizontal velocity components. The comparison of turbulent and tidal horizontal velocities was made by isolating the semidiurnal signal, associated with lowest horizontal and vertical modes, in spectral space. The remaining modes then correspond to the turbulent motions. An $x-z$ cross section of the $x$ component of velocity during the wave breaking phase of the simulation is shown in Fig. A1a. Primary wave and turbulent motions are separated in Figs. A1b,c, respectively, to illustrate the relative contributions of each component. The overall flow is clearly dominated by the primary wave.

The ratio of turbulent velocity fluctuations to mean flow (primary wave) velocity defines a turbulence intensity that provides a quantitative measure of the validity of the Taylor hypothesis (Fig. A2). Excluding the initial period of onset of instability and focusing instead on the turbulent phase of the wave breaking between $2 \times 10^{4}$ and $1 \times 10^{5} \mathrm{~s}$, we find that the volume-mean turbulence intensity is less than 0.25 during $82 \%$ of the time and greater than one during $6 \%$ of the time. Therefore, most of the time the advection is clearly dominated by the mean flow. We also verified that advection is primarily in the horizontal direction. 
To enable a direct validation of the Taylor hypothesis for this flow, time series with high temporal sampling equal to $15 \mathrm{~s}$ were recorded at evenly distributed horizontal moorings positioned throughout the numerical domain. Time series at each numerical mooring were then analyzed in the same fashion as the observations and were used to infer spatial horizontal spectra. These spectra were then compared to horizontal spectra computed directly from the temperature spatial distribution. The spectra at one typical mooring are shown in Fig. A3. The two spectra agree within the wavenumber range $7 \times 10^{-3}$ and $2 \times 10^{-2}$ $\mathrm{cpm}$, in the interval corresponding to the stratified inertial subrange with a slope close to $-5 / 3$. The dissipation rate $\epsilon$ was also inferred.

\section{REFERENCES}

Alisse, J. R., and C. Sidi, 2000: Experimental probability density functions of small-scale fluctuations in the stably stratified atmosphere. J. Fluid Mech., 402, 137-162.

Bénielli, D., and J. Sommeria, 1996: Excitation of internal waves and stratified turbulence by parametric instability. Dyn. Atmos. Oceans, 23, 335-343.

Bonnin, J., H. van Haren, P. Hosegood, and G.-J. A. Brummer, 2006: Burst resuspension of seabed material at the foot of the continental slope in the Rockall channel. Mar. Geol., 226, 167-184.

Bouruet-Aubertot, P., J. Sommeria, and C. Staquet, 1996: Stratified turbulence produced by internal wave breaking. Dyn. Atmos. Oceans, 23, 371-378.

$\ldots,-$, B. Le Cann, and C. R. Koudella, 2004: Intermittency of vertical density gradients at finescale and link with mixing processes. Deep-Sea Res., 51, 2919-2941.

Carter, G. S., and M. C. Gregg, 2002: Intense, variable mixing near the head of Monterey Submarine Canyon. J. Phys. Oceanogr., 32, 3145-3165.

D'Asaro, E. A., and R. C. Lien, 2000a: Lagrangian measurements of waves and turbulence in stratified flows. J. Phys. Oceanogr., 30, 641-655.

—, and 2000b: The wave-turbulence transition for stratified flows. J. Phys. Oceanogr., 30, 1669-1678.

Dugan, J. P., W. D. Morris, and B. S. Okawa, 1986: Horizontal wavenumber distribution of potential energy in the ocean. J. Geophys. Res., 91 (C11), 12 993-13 000

Ewart, T. E., 1976: Observations from straightline isobaric runs of SPURV. Proc. IAPSO/IAMAP PSII, Edinburgh, United Kingdom, Joint Oceanography Assembly, 1-18.

Frehlich, R., Y. Meilier, and M. L. Jensen, 2008: Measurements of boundary layer profiles with in situ sensors and Doppler radar. J. Atmos. Ocean. Technol., 25, 1328-1340.

Frisch, U., 1995: Turbulence: The Legacy of A. N. Kolmogorov. Cambridge University Press, 296 pp.

Garrett, C. J. R., and W. H. Munk, 1972: Space time scales of internal waves. Geophys. Fluid Dyn., 3, 225-264.

Gregg, M. C., 1977: Variations in the intensity of small-scale mixing in the main thermocline. J. Phys. Oceanogr., 7, 436-454.

_ 1987: Diapycnal mixing in the thermocline: A review. J. Geophys. Res., 92 (C5), 5249-5286.

Holbrook, W. S., and I. Fer, 2005: Oceanic internal wave spectra inferred from seismic reflection transect. Geophys. Res. Lett., 32, L15604, doi:10.1029/2005GL023733.
Ivey, G. N., K. B. Winters, and J. R. Joseff, 2008: Density stratification, turbulence, but how much mixing? Annu. Rev. Fluid Mech., 40, 169-184.

Katz, E. J., and M. G. Briscoe, 1979: Vertical coherence of the internal wave field from towed sensors. J. Phys. Oceanogr., 9, $518-530$.

Klymak, J. M., and J. N. Moum, 2007a: Oceanic isopycnal slope spectra. Part I: Internal waves. J. Phys. Oceanogr., 37, 1215-1231. , and - 2007b: Oceanic isopycnal slope spectra. Part II: Turbulence. J. Phys. Oceanogr., 37, 1232-1245.

, R. Pinkel, and L. Rainville, 2008: Direct breaking of the internal tide near topography: Kaena Ridge, Hawaii. J. Phys. Oceanogr., 38, 380-399.

Lelong, M.-P., and T. J. Dunkerton, 1998a: Inertia-gravity wave breaking in three dimensions. Part I: Convectively stable waves. J. Atmos. Sci., 55, 2473-2488.

, and $\_$, 1998b: Inertia-gravity wave breaking in three dimensions. Part II: Convectively unstable waves. J. Atmos. Sci., 55, 2489-2501

Lien, R.-C., and E. A. D'Asaro, 2006: Measurements of turbulent kinetic energy dissipation rate with a Lagrangian float. J. Atmos. Oceanic Technol., 23, 964-976.

— M. J. McPhaden, and M. C. Gregg, 1996: High-frequency internal waves in the upper central equatorial Pacific and their possible relationship to deep-cycle turbulence. J. Phys. Oceanogr., 26, 581-600.

Lindborg, E., 2006: The energy cascade in a strongly stratified fluid. J. Fluid Mech., 550, 207-242.

McKean, R. C., and T. E. Ewart, 1974: Temperature spectra in the deep ocean off Hawaii. J. Phys. Oceanogr., 4, 191-199.

McPhaden, M. J., and H. Peters, 1992: Diurnal cycle of internal wave variability in the equatorial Pacific Ocean: Results from moored observations. J. Phys. Oceanogr., 22, 1317-1329.

Munk, W., and C. Wunsch, 1998: Abyssal recipes II: Energetics of tidal and wind mixing. Deep-Sea Res. I, 45, 1977-2010.

Nastrom, G. D., and K. S. Gage, 1985: A climatology of atmospheric wave number spectra of wind and temperature observed by commercial aircraft. J. Atmos. Sci., 42, 950-960.

Osborn, T., 1980: Estimates of the local rate of vertical diffusion from dissipation measurements. J. Phys. Oceanogr., 10, 83-89.

Pinton, J.-F., and R. Labbé, 1994: Correction to the Taylor hypothesis in swirling flows. J. Phys. II, 4, 1461-1468.

Polzin, K., E. Kunze, J. M. Toole, and R. W. Schmitt, 2003: The partition of finescale energy into internal waves and subinertial motions. J. Phys. Oceanogr., 33, 234-248.

Riley, J. J., and S. M. de Bruyn Kops, 2003: Dynamics of turbulence strongly influenced by buoyancy. Phys. Fluids, 15, 2047-2059. , and E. Lindborg, 2008: Stratified turbulence: A possible interpretation of some geophysical turbulence measurements. J. Atmos. Sci., 65, 2416-2424.

Seuront, L., and F. G. Schmitt, 2004: Eulerian and Lagrangian properties of biophysical intermittency. Geophys. Res. Lett., 31, L03306, doi:10.1029/2003GL018185.

Sreenivasan, K. R., 1996: The passive scalar spectrum and the Obukhow-Corrsin constant. Phys. Fluids, 8, 189-196.

Tennekes, H., and J. L. Lumley, 1972: A First Course in Turbulence. MIT Press, 300 pp.

van Haren, H., R. Groenewegen, M. Laan, and B. Koster, 2001: A fast and accurate thermistor string. J. Atmos. Oceanic Technol., 18, 256-265.

Zaman, K. B. M. Q., and A. K. F. M. Hussain, 1981: Taylor hypothesis and large-scale coherent structures. J. Fluid Mech., 112, 379-396. 